\title{
FORS2/VLT survey of Milky Way globular clusters
}

\section{Description of the method for derivation of metal abundances in the optical and application to NGC 6528, NGC 6553, M 71, NGC 6558, NGC 6426, and Terzan $8^{\star}, \star \star$}

\author{
B. Dias ${ }^{1,2}$, B. Barbuy ${ }^{1}$, I. Saviane ${ }^{2}$, E. V. Held ${ }^{3}$, G. S. Da Costa ${ }^{4}$, S. Ortolani ${ }^{3,5}$, S. Vasquez ${ }^{2,6}$, \\ M. Gullieuszik ${ }^{3}$, and D. Katz ${ }^{7}$
}

\author{
1 Universidade de São Paulo, Dept. de Astronomia, Rua do Matão 1226, 05508-090 São Paulo, Brazil \\ e-mail: bdias@astro.iag.usp.br \\ 2 European Southern Observatory, 3107 Alonso de Cordova, Santiago, Chile \\ 3 INAF, Osservatorio Astronomico di Padova, Vicolo dell’Osservatorio 5, 35122 Padova, Italy \\ ${ }^{4}$ Research School of Astronomy \& Astrophysics, Australian National University, Mount Stromlo Observatory, via Cotter Road, \\ Weston Creek, ACT 2611, Australia \\ 5 Università di Padova, Dipartimento di Astronomia, Vicolo dell'Osservatorio 2, 35122 Padova, Italy \\ 6 Instituto de Astrofisica, Facultad de Fisica, Pontificia Universidad Catolica de Chile, Casilla 306 Santiago 22, Chile \\ 7 GEPI, Observatoire de Paris, CNRS, Université Paris Diderot, 5 place Jules Janssen, 92190 Meudon, France
}

Received 14 April 2014 / Accepted 12 September 2014

\begin{abstract}
Context. We have observed almost one third of the globular clusters in the Milky Way, targeting distant and/or highly reddened objects, as well as a few reference clusters. A large sample of red giant stars was observed with FORS2 at VLT/ESO at $R \sim 2000$. The method for deriving stellar parameters is presented with application to six reference clusters.

Aims. We aim at deriving the stellar parameters effective temperature, gravity, metallicity, and alpha-element enhancement as well as the radial velocity to confirm the membership of individual stars in each cluster. We analyse the spectra collected for the reference globular clusters NGC $6528([\mathrm{Fe} / \mathrm{H}] \sim-0.1), \mathrm{NGC} 6553([\mathrm{Fe} / \mathrm{H}] \sim-0.2), \mathrm{M} 71([\mathrm{Fe} / \mathrm{H}] \sim-0.8), \mathrm{NGC} 6558([\mathrm{Fe} / \mathrm{H}] \sim-1.0)$, NGC $6426([\mathrm{Fe} / \mathrm{H}] \sim-2.1)$, and Terzan $8([\mathrm{Fe} / \mathrm{H}] \sim-2.2)$. They cover the full range of globular cluster metallicities, and are located in the bulge, disc, and halo.

Methods. Full spectrum-fitting techniques were applied by comparing each target spectrum with a stellar library in the optical region at 4560-5860 ̊. We employed the library of observed spectra MILES, and the Coelho synthetic library. We validated the method by recovering the known atmospheric parameters for 49 well-studied stars that cover a wide range in the parameter space. We adopted as final stellar parameters (effective temperatures, gravities, metallicities) the average of results using the observed and synthetic spectral libraries.

Results. We identified 4 member stars in NGC 6528, 13 in NGC 6553, 10 in M 71, 5 in NGC 6558, 5 in NGC 6426, and 12 in Terzan 8. Radial velocities, $T_{\text {eff }}, \log (g),[\mathrm{Fe} / \mathrm{H}]$, and alpha-element enhancements were derived. We derived $\left\langle v_{\text {helio }}\right\rangle=-242 \pm 11 \mathrm{~km} \mathrm{~s}{ }^{-1},[\mathrm{Fe} / \mathrm{H}]=$ $-2.39 \pm 0.04,[\mathrm{Mg} / \mathrm{Fe}]=0.38 \pm 0.06$ for NGC 6426 from spectroscopy for the first time.

Conclusions. The method proved to be reliable for red giant stars observed with resolution $R \sim 2000$, yielding results compatible with high-resolution spectroscopy. The derived $\alpha$-element abundances show $[\alpha / \mathrm{Fe}]$ vs. $[\mathrm{Fe} / \mathrm{H}]$ consistent with that of field stars at the same metallicities.
\end{abstract}

Key words. stars: abundances - stars: kinematics and dynamics - stars: Population II - globular clusters: general Galaxy: stellar content

\section{Introduction}

Stellar metallicities and abundances are best derived from high spectral resolution and high signal-to-noise $(\mathrm{S} / \mathrm{N})$ data. Cayrel (1988) showed that a higher resolution carries more information than a higher $\mathrm{S} / \mathrm{N}$. These types of data require substantial telescope time, however. For this reason, very large samples of stellar spectra have been gathered in recent years, or are planned

\footnotetext{
* Based on observations collected at the European Southern Observatory/Paranal, Chile, under programmes 077.D-0775(A) and 089.D-0493(B).

$\star \star$ Figures 14-18, Tables 3, 4, and 6 are available in electronic form at http://www . aanda.org
}

to be collected in the near future, with multi-object low- and medium-resolution instruments. A few examples are the Sloan Digital Sky Survey (SDSS, York et al. 2000), at a resolution $R \sim$ 1800, the Radial Velocity Experiment survey (RAVE, Steinmetz et al. 2006) of $R \sim 7500$ in the CaT region, other large ongoing surveys such as LAMOST at the Guoshoujing telescope (GSJT, Wu et al. 2011) of $R \sim 2000$, and future surveys such as Gaia (Perryman et al. 2001). Large data sets of low- and mediumresolution spectra are available for extragalactic stars, such as presented in Kirby et al. (2009). A few recent surveys are able to use medium- and high-resolution spectra focused on specific targets such as provided by APOGEE ( $R \sim 22500$, Mészáros et al. 2013), Gaia-ESO using the FLAMES-GIRAFFE spectrograph 
$(R \sim 22000)$ at the Very Large Telescope (VLT, Gilmore et al. 2012), and HERMES ( $R \sim 28000$ or 45000 , Wylie-de Boer \& Freeman 2010) at the AAT. More complete reviews of available, ongoing, and future surveys, as well as automated methods for stellar parameter derivation can be found in Allende Prieto et al. (2008), Lee et al. (2008), Koleva et al. (2009), Mészáros et al. (2013), and Wu et al. (2011), among others.

Most analyses of medium- to low-resolution spectra employ the least squares ( $\chi^{2}$ minimization), or euclidian distance, also called minimum distance method, for example, the Université de Lyon Spectroscopic Analysis Software (ULySS, Koleva et al. 2009), and the k-means clustering described in Sánchez Almeida \& Allende Prieto (2013).

In the present work we analyse spectra in the optical in the range 4560-5860 $\AA$, obtained at the FORS2/VLT at a resolution $R \sim 2000$ and carry out a full spectrum fitting. This spectral region, in particular from $\mathrm{H}_{\beta}$ to $\mathrm{Na}$ I lines, is sensitive to metallicity, temperature, and gravity through the $\mathrm{MgH}$ molecular bands (as part of the $\mathrm{Mg}_{2}$ index). Also, it includes the Lick indices Fe5270, Fe5335, and $\mathrm{Mg}_{2}$, which are common Fe and Mg abundance indicators (Katz et al. 2011; Cayrel et al. 1991; Faber et al. 1985; Worthey et al. 1994).

The same sample was observed in the near-infrared (CaT), as presented in Saviane et al. (2012), Da Costa et al. (2009), and Vasquez et al. (in prep.), where two of the Ca II triplet lines were used to derive velocities and metallicities. A comparison of their results with ours shows good consistency, as we discuss below.

In this work we study six reference globular clusters, spanning essentially the full range of metallicities of globulars: the metal-poor halo clusters NGC 6426 and Terzan 8 $([\mathrm{Fe} / \mathrm{H}] \sim-2.1$ and -2.2$)$, the moderately metal-poor NGC 6558 $([\mathrm{Fe} / \mathrm{H}] \sim-1.0)$ in the bulge, the template disc metal-rich cluster M 71 (NGC 6838, [Fe/H] -0.7), and the metal-rich bulge clusters NGC 6528 and NGC $6553([\mathrm{Fe} / \mathrm{H}] \sim-0.1$ and -0.2$)$.

These reference clusters are analysed with the intent of testing and improving the method, and verifying the metallicity range of applicability of each library of template spectra. In all cases, member stars and surrounding field stars are analysed. For some of these clusters previous high-resolution spectroscopic and photometric data of a few member stars are available.

The minimum distance method was adopted by Cayrel et al. (1991) by measuring residuals in each of the stellar parameters effective temperature, gravity, and metallicity; the method required the input of reference parameters. In the present work, we adopt the code ETOILE (Katz et al. 2011), which uses the minimum distance method, where the reliability and coverage of $T_{\text {eff }}, \log (g),[\mathrm{Fe} / \mathrm{H}]$, and $[\alpha / \mathrm{Fe}]$ of the template stars are important to find well-founded parameters for the target stars. We adopted two different libraries of spectra, the MILES ${ }^{1}$ library of low-resolution spectra $(R \sim 2000)$ and the grid of synthetic spectra computed by Coelho et al. $(2005)^{2}$.

In Sect. 2 the observations are described. In Sect. 3 the method of stellar parameter derivation is detailed. In Sect. 4 the method is applied to six clusters as a validation of the procedures. In Sect. 5 the results are discussed, and in Sect. 6 a summary is given.

\footnotetext{
1 http://miles.iac.es/

2 http://www.mpa-garching.mpg.de/PUBLICATIONS/DATA/

SYNTHSTELLIB/synthetic_stellar_spectra.html
}

Table 1. Telescope and spectrograph.

\begin{tabular}{l|c}
\hline \hline \multicolumn{2}{c}{ Observing information } \\
\hline Telescope & Antu/UT1-VLT at ESO \\
Instrument & FORS2 \\
Grism & $1400 \mathrm{~V}$ \\
FoV & $6.8 \times 6.8$ \\
Pixel scale & $0.25^{\prime \prime} / \mathrm{pixel}$ \\
Slit width & $0.53 \mathrm{~mm}$ \\
Spec. resolution & $R=2000$ \\
Dispersion & $0.6 \AA$ A $/ \mathrm{pix}$ \\
\hline
\end{tabular}

\section{Observations and data reduction}

We observed with FORS2 at VLT/ESO (Appenzeller et al. 1998) 17 red giant stars in the globular cluster NGC 6528, 17 in NGC 6553, 12 in M 71, 17 in NGC 6558, 10 in NGC 6426, and 13 in Terzan 8, under projects 077.D-0775(A) and 089.D0493(B). Table 1 summarizes the observation setup. Pre-images were taken using filters Johnson-Cousins $V$ and $I$ to select only stars in the red giant branch (RGB) brighter than the red clump (RC) level. Zero points in colours and magnitudes were fitted to match isochrones with parameters from Table 2 (see colourmagnitude diagrams, CMDs, in Fig. 1). We selected stars covering the whole interval in colour of the RGB, and when possible, tried to avoid asymptotic giant branch (AGB) stars. These stars are spatially distributed as shown in Fig. 2, partly because of the slitlet configuration. Cluster parameters and log of observations are given in Table 2. The list of individual stars, their coordinates, and VI magnitudes from our FORS2 observations are given in Table 3 .

The spectra were taken using the grism $1400 \mathrm{~V}$, centred on $5200 \AA$, covering the range $4560-5860 \AA$, with a resolution of $R \sim 2000$. Figure 3 illustrates the spectra of a metal-poor and a metal-rich red giant star, where many of the strongest lines are indicated.

The spectra were reduced using the pipeline ${ }^{3}$ esorex/FORS2 with default parameters for bias and flatfield correction, spectra extraction, and wavelength calibration. The only modification relative to default parameters was a list of skylines, since the default list had only one line. The wavelength calibration proved to be satisfactory with this line list. A last step in the reduction procedure was a manual removal of cosmic rays.

\section{Stellar parameter derivation}

\subsection{Radial velocities}

Radial velocities were measured using the code ETOILE through cross-correlation with a template spectrum from the chosen library. We tested the results by measuring radial velocities using fxcor at IRAF (cross-correlation), and rvidlines at IRAF (using the wavelength of $\mathrm{MgI}$ triplet lines as a reference). The derived velocities are consistent, therefore we also used ETOILE to determine radial velocities. The mean FWHM of the arc lines is $2.36 \pm 0.04 \AA\left(125 \mathrm{~km} \mathrm{~s}^{-1}\right)$. This leads to a radial velocity uncertainty of $\sim 13 \mathrm{~km} \mathrm{~s}^{-1}$. Heliocentric radial velocities for each star can be found in Table 3, where the last column refers to the values measured from the CaII triplet (CaT) lines in the near infrared by Saviane et al. (2012) for member stars for NGC 6528, NGC 6553, M 71, and NGC 6558, and by

\footnotetext{
http://www.eso.org/sci/software/pipelines/
} 
B. Dias et al.: FORS2/VLT survey of Milky Way globular clusters. I.

Table 2. Log of observations and cluster parameters from the literature.

\begin{tabular}{|c|c|c|c|c|c|c|}
\hline Parameter & NGC 6528 & NGC 6553 & M 71 & NGC 6558 & NGC 6426 & Terzan 8 \\
\hline $\begin{array}{c}\text { Date of obs. } \\
\text { UT } \\
\tau\end{array}$ & $\begin{array}{c}29.05 .2006 \\
08: 36: 22 \\
149.4 \mathrm{~s}\end{array}$ & $\begin{array}{c}29.05 .2006 \\
08: 57: 50 \\
79.4 \mathrm{~s}\end{array}$ & $\begin{array}{c}29.05 .2006 \\
09: 14: 32 \\
17.2 \mathrm{~s}\end{array}$ & $\begin{array}{c}29.05 .2006 \\
06: 55: 32 \\
148.3 \mathrm{~s}\end{array}$ & $\begin{array}{c}13.07 .2012 \\
02: 31: 12 \\
500.0 \mathrm{~s}\end{array}$ & $\begin{array}{c}12.07 .2012 \\
07: 47: 29.346 \\
360 \mathrm{~s}\end{array}$ \\
\hline $\begin{array}{c}\text { RA } \\
\text { Dec } \\
\text { age } \\
{[\mathrm{Fe} / \mathrm{H}]} \\
{[\mathrm{Mg} / \mathrm{Fe}]^{a} \text { or }[\alpha / \mathrm{Fe}]^{b}} \\
E(B-V) \\
(m-M)_{\mathrm{V}} \\
R_{\text {Sun }} \\
R_{\mathrm{GC}} \\
\left\langle v_{\text {helio }}\right\rangle \\
r_{\text {core }} \\
r_{\text {tidal }} \\
r_{\text {half-light }}\end{array}$ & $\begin{array}{c}18^{\mathrm{h}} 04^{\prime} 49.64^{\prime \prime} \\
-30^{\circ} 03^{\prime} 22.6^{\prime \prime} \\
13 \mathrm{Gyr}^{(1)} \\
-0.11 \mathrm{dex} \\
0.24^{(b, 5)} \\
0.54 \\
16.17 \\
7.9 \mathrm{kpc} \\
0.6 \mathrm{kpc} \\
206.6 \pm 1.4 \mathrm{~km} \mathrm{~s}^{-1} \\
0.13^{\prime} \\
4.11^{\prime} \\
0.38^{\prime}\end{array}$ & $\begin{array}{c}18^{\mathrm{h}} 09^{\prime} 17.60^{\prime \prime} \\
-25^{\circ} 54^{\prime} 31.3^{\prime \prime} \\
13 \mathrm{Gyr}^{(1)} \\
-0.18 \mathrm{dex} \\
0.26 \mathrm{dex}^{(b, 6)} \\
0.63 \\
15.83 \\
6.0 \mathrm{kpc} \\
2.2 \mathrm{kpc} \\
-3.2 \pm 1.5 \mathrm{~km} \mathrm{~s}^{-1} \\
0.53^{\prime} \\
7.66^{\prime} \\
1.03^{\prime}\end{array}$ & $\begin{array}{c}19^{\mathrm{h}} 53^{\prime} 46.49^{\prime \prime} \\
+18^{\circ} 46^{\prime} 45.1^{\prime \prime} \\
11.00 \pm 0.38 \mathrm{Gyr}^{(2)} \\
-0.78 \mathrm{dex} \\
0.19 \pm 0.04^{(a, 7)}, 0.40^{(b, 5)} \\
0.25 \\
13.80 \\
4.0 \mathrm{kpc} \\
6.7 \mathrm{kpc} \\
-22.8 \pm 0.2 \mathrm{~km} \mathrm{~s}^{-1} \\
0.63^{\prime} \\
8.90^{\prime} \\
1.67^{\prime}\end{array}$ & $\begin{array}{c}18^{\mathrm{h}} 10^{\prime} 17.60^{\prime \prime} \\
-31^{\circ} 45^{\prime} 50.0^{\prime \prime} \\
14 \mathrm{Gyr}^{(3)} \\
-0.97 \pm 0.15 \mathrm{dex}^{(3)} \\
0.24^{(a, 3)} \\
0.44 \\
15.70 \\
7.4 \mathrm{kpc} \\
1.0 \mathrm{kpc} \\
-197.3 \pm 4 \mathrm{~km} \mathrm{~s}^{-1(3)} \\
0.03^{\prime} \\
9.49^{\prime} \\
2.15^{\prime}\end{array}$ & $\begin{array}{c}17^{\mathrm{h}} 44^{\prime} 54.65^{\prime \prime} \\
+03^{\circ} 10^{\prime} 12.5^{\prime \prime} \\
13.0 \pm 1.5 \mathrm{Gyr}^{(4)} \\
-2.15 \mathrm{dex} \\
0.4^{(b, 4)} \\
0.36 \\
17.68 \\
20.6 \mathrm{kpc} \\
14.4 \mathrm{kpc} \\
-162.0 \mathrm{~km} \mathrm{~s}^{-1} \\
0.26^{\prime} \\
13.03^{\prime} \\
0.92^{\prime}\end{array}$ & $\begin{array}{c}19^{\mathrm{h}} 41^{\prime} 44.41^{\prime \prime} \\
-33^{\circ} 59^{\prime} 58.1^{\prime \prime} \\
13.00 \pm 0.38 \mathrm{Gyr}^{(2)} \\
-2.16 \mathrm{dex} \\
0.47 \pm 0.09^{(a, 8)} \\
0.12 \\
17.47 \\
26.3 \mathrm{kpc} \\
19.4 \mathrm{kpc} \\
130.0 \mathrm{~km} \mathrm{~s}^{-1} \\
1.00 \\
3.98 \\
0.95\end{array}$ \\
\hline
\end{tabular}

Notes. The main reference is Harris (1996, 2010 edition), when not indicated otherwise. ${ }^{(1)}$ Zoccali et al. (2001); ${ }^{(2)}$ VandenBerg et al. (2013); (3) Barbuy et al. (2007); (4) Dotter et al. (2011); (5) Carretta et al. (2010); ${ }^{(6)}$ Cohen et al. (1999); ${ }^{(7)}$ Meléndez \& Cohen (2009); (8) Carretta et al. (2014).
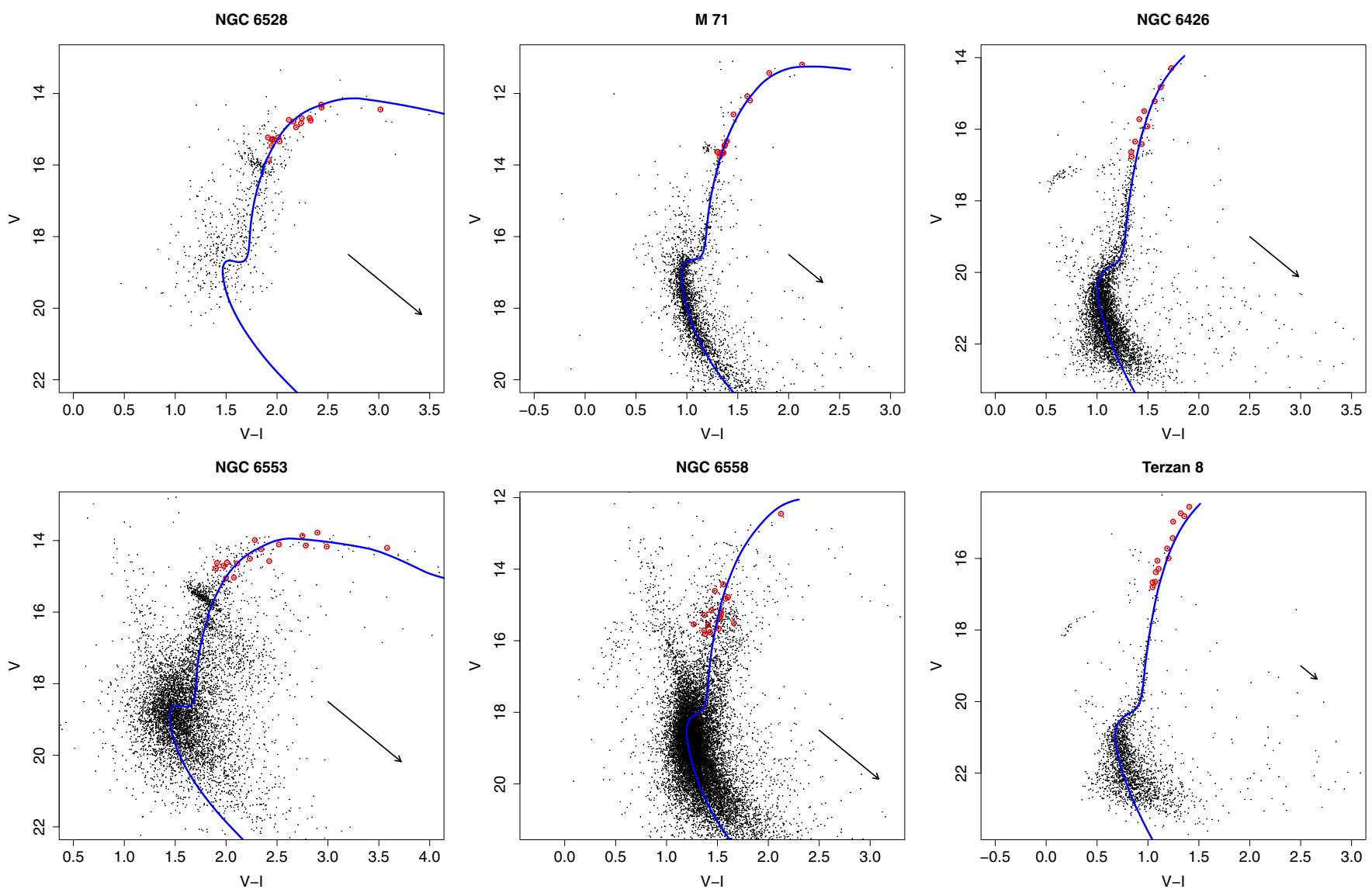

Fig. 1. Colour-magnitude diagrams of all clusters. The left panels show metal-rich clusters, middle panels are intermediate-metallicity clusters, and right panels correspond to the more metal-poor ones. All stars within $2 \times r_{\text {half-light }}$ are plotted, without any cleaning procedure. Dartmouth isochrones with literature parameters (Table 2) are overplotted. Selected RGB stars for spectroscopic observations are plotted in red. Reddening vectors are shown in each CMD based on $E(B-V)$ listed in Table 2.

Vasquez et al. (in prep.) for NGC 6426 and Terzan 8. The present radial velocity values and those from the CaT line region agree well and a few exceptions are stars 8 of NGC 6558,2, 10 of M 71, among others. A possible explanation for this might be an imperfectly centred source in the slit in some cases, as suggested by Katz et al. (2011) from using CFHT-MOS. Average values for member stars in each cluster are presented in Fig. 4, where our results are compared with CaT results (Saviane et al. 2012 and Vasquez et al., in prep.), and with the Harris catalogue (1996, 2010 edition). The error bars from the literature are smaller than 

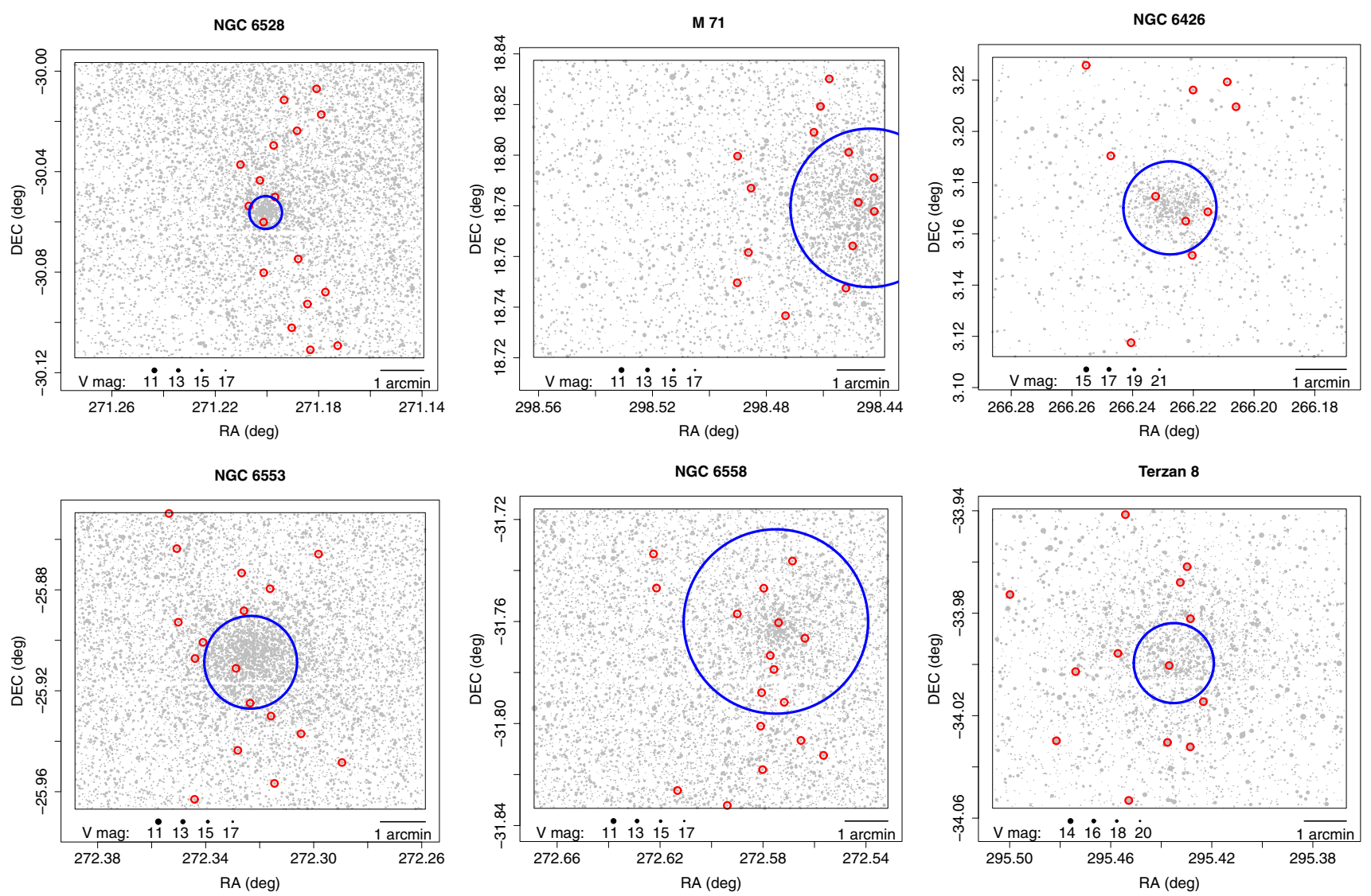

Fig. 2. Sky map of all clusters. The panels show the same as in Fig. 1. Only the brightest stars are shown, and the size of the dots scales with the star magnitudes, as indicated in each plot. Selected RGB stars for spectroscopic observations are plotted in red. The blue circle corresponds to the half-light radius of each cluster from Table 2.

the empty circles that represent the literature $\mathrm{v}_{\text {helio, }}$, except for NGC 6426, as can be seen in Fig. 4. Our results agree well with both references. In particular, the radial velocity measured for NGC 6426 agrees well between this work and the CaT results based on individual member stars, but it is only compatible with the literature value within $3 \sigma$. The explanation is that the only work that measured radial velocities for this cluster was based on integrated light from photographic plates (Hesser et al. 1986). Therefore, our radial velocity derivation for NGC 6426 is more reliable.

\subsection{Atmospheric parameters}

Full spectrum fitting with minimum distance method was employed, using the ETOILE code described in Katz et al. (2011) and Katz (2001). We applied the calculations to the wavelength region $4560-5860 \AA$, similarly to the procedure described in Katz et al. (2011).

We automatically derived the atmospheric parameters $\left(T_{\mathrm{eff}}\right.$, $\log (g),[\mathrm{Fe} / \mathrm{H}],[\alpha / \mathrm{Fe}])$ of a stellar spectrum by comparing the target spectrum with each library spectrum, thus covering a wide range of atmospheric parameters. In each comparison, ETOILE fits the template spectrum to the observed spectrum. Mathematically, ETOILE solves by least squares for the polynomial by which to multiply the template spectrum to minimize the differences with the observed spectrum (see Eqs. (1) and (2)). The aim of these operations is to compensate for the differences between the template and observed spectra that are not from stellar origin such as flux level and normalisation, instrumental profile, and interstellar reddening. In particular, concerning this last point, no explicit reddening is applied to the template. The differential reddening correction is included in the fitting of the template to the observed spectrum,

$$
S=\sqrt{\sum_{i=0}^{n}\left\{F_{\text {obs }}(i)-\left[\sum_{j=0}^{m} u_{j} \cdot\left(\lambda(i)-\lambda_{\text {central }}\right)^{j}\right] \cdot F_{\text {templ }}(i)\right\}^{2}},
$$

where $n$ is the number of pixels in the analysed spectrum, $F_{\text {obs }}(i)$ and $F_{\text {templ }}(i)$ are the fluxes of the analysed and the template spectra pixel by pixel (i.e. lambda by lambda), $m$ is the order of the polynomial that multiplies $F_{\text {templ }}(i), u_{j}$ are the coefficients, and $\lambda_{\text {central }}=[\lambda(0)+\lambda(n)] / 2$. Equation (1) is minimized to find the multiplicative polynomial that minimizes the differences in flux between observed and template spectra by solving the $m+1$ Equations below. We adopted $m=4$.

$\frac{\partial S}{\partial u_{j}}=0$, where $j \in\{0, \ldots, m\}$.

After determining the polynomial that minimizes the difference between each template and the observed spectrum, as defined by Eq. (1), templates were ranked in order of increasing $S$ and the parameters of the top $N$ templates were averaged out to produce the final results. Determination of the best-fit value of $N$ is discussed in Sect. 3.2.2. This is called the similarity method and was introduced by Katz et al. (1998). For a more detailed explanation see Katz (2001).

Before running the code, two important steps are necessary: convolving all the library spectra to the same resolution of the target spectra, and correcting for radial velocities $v_{r}$. Convolution calculations were performed for the library spectra using the task 
B. Dias et al.: FORS2/VLT survey of Milky Way globular clusters. I.
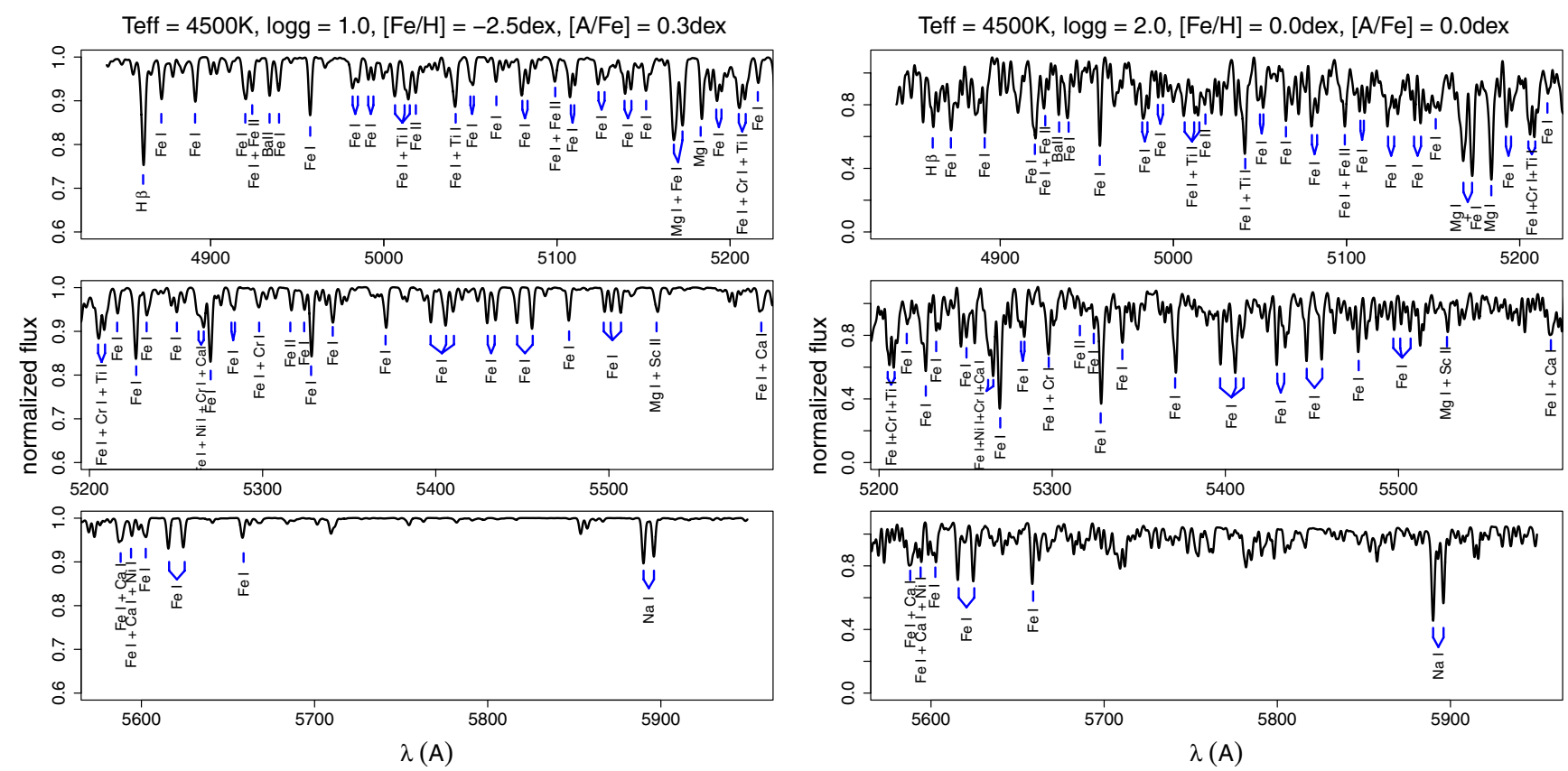

Fig. 3. Most important atomic lines in each strong spectral feature with FORS2 resolution $(R \sim 2000)$. The most important molecular band in this region is $\mathrm{MgH}$ around $\lambda=5165 \AA$, on top of the $\mathrm{Mg}$ I triplet lines. The left panel shows a metal-poor red giant star from the library of Coelho et al. (2005), the right panel shows a metal-rich. The left panels were zoomed in the $y$-axis direction for better visualization.

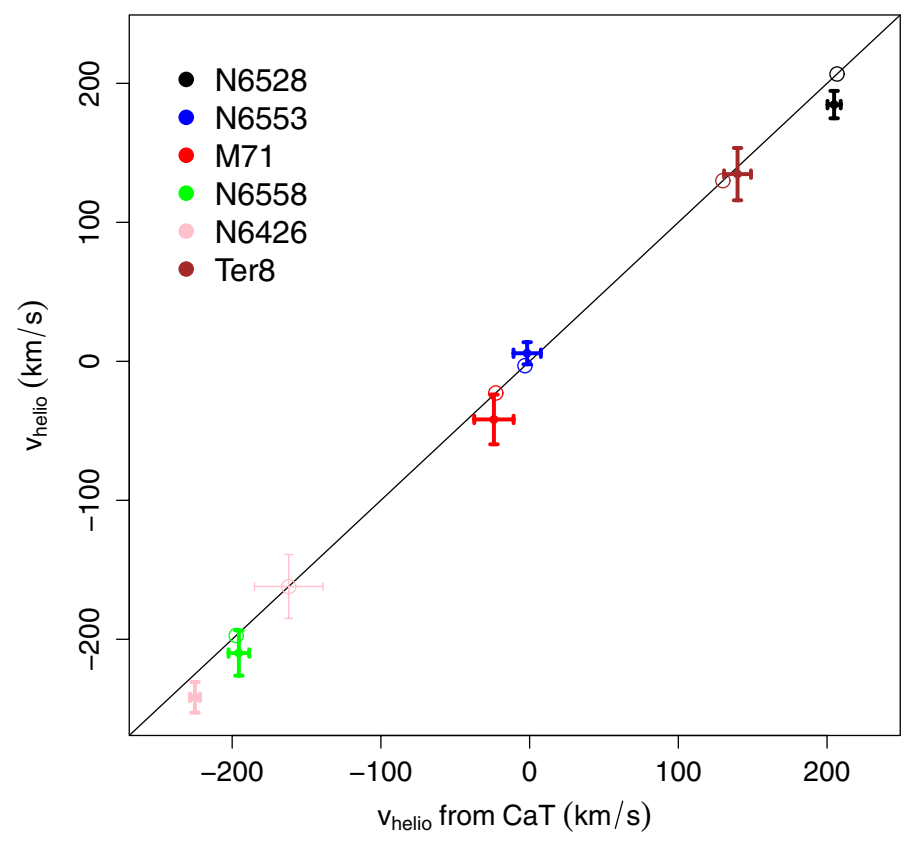

Fig. 4. Average of heliocentric radial velocities of member stars (see details in Sect. 4.3 and values in Table 3) for each globular cluster. Our results are plotted against those from CaT spectroscopy (Saviane et al. 2012) and Vasquez et al. (in prep.) which match well. The error bars are the standard deviation of the average. A one-to-one line is plotted to guide the eye, while radial velocities from Harris (1996, 2010 edition) are overplotted as empty circles.

GAUSS in IRAF. The code ETOILE measures the radial velocities by comparing them with template spectra from the library, a reliable way of measuring $v_{r}$ in each observed spectrum and correct it.

Figure 5 shows six examples of spectral fitting: for a metalpoor (Terzan8_11), a metal-rich (NGC 6528_11), and an intermediate metallicity star (NGC 6558_7) using the templates from Coelho and MILES. The template stars that best fit these cluster stars of the available spectra from the MILES library are BD+060648, HD 161074, and HD 167768. For Coelho the best templates have the following parameters: $\left(T_{\text {eff }}, \log (g)\right.$, $[\mathrm{Fe} / \mathrm{H}])=(5000 \mathrm{~K}, 2.5,-2.0),(3500 \mathrm{~K}, 0.0,-1.5)$, and $(5000 \mathrm{~K}$, $3.0,-1.0)$, respectively. The residuals shown at the bottom of each panel indicate that the metal-poor target spectrum is similar to the template spectrum within $2 \%$ for both libraries, except for a few strong features. The residuals for the metal-rich star show a similarity between target and template spectra of $5 \%$ for MILES and of $7 \%$ for Coelho, except for the boundaries $\lambda \gtrsim 5700 \AA$ and $\lesssim 4700 \AA$, and for a few strong features. For the intermediate-metallicity star the residuals present a sigma of $3 \%$ for both libraries with a few stronger features that vary by more than $3 \%$. These differences between the spectra are reflected in the atmospheric parameters, and they are compensated for by taking the average of parameters of the most similar spectra. For Terzan8_11, eight MILES spectra were close enough and were averaged, for NGC 6528_11, 21 MILES spectra were considered, and for NGC 6558_7 we found eight stars. For all cases we considered ten templates on Coelho library. Details on the criterion used to select the number of template spectra are discussed in Sect. 3.2.2.

\subsubsection{Stellar libraries}

The core of the atmospheric parameter derivation is the choice of a stellar library. There are two classes of stellar libraries: based on observed or based on synthetic spectra. The real spectra are more reliable, but their drawback are abundances typical of nearby stellar populations. The synthetic libraries have no noise and cover a large and uniform atmospheric parameter space, but completeness of atomic and molecular line lists are still limited and there are uncertainties on oscillator strengths and assumptions on atmospheric models, such as 1D and local thermodynamical equilibrium. For these reasons, it is useful to 

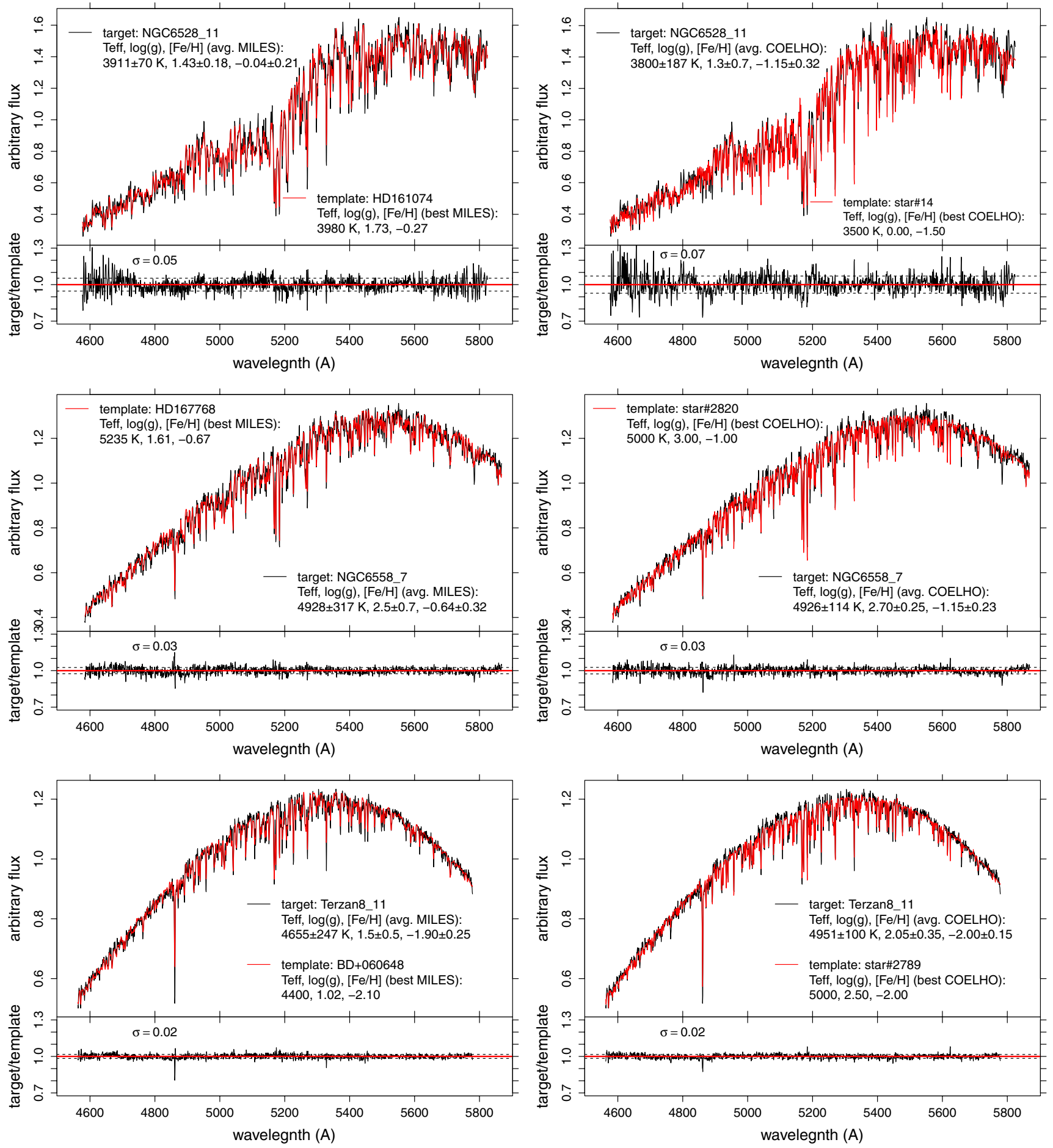

Fig. 5. Examples of spectral fitting carried out with ETOILE for a metal-rich (NGC 6528_11), an intermediate-metallicity (NGC 6558_7), and a metal-poor (Terzan8_11) star in the upper, middle, and bottom panels, respectively. The left panels represent the best fits using MILES template spectra, the right panel the best fits using Coelho spectra. Stellar spectrum (black line) is overplotted by the template spectrum (red line) that best fits it. The residuals of each fit are presented below each stellar spectrum. The match between the spectra is made following the procedures explained in Sect. 3.2. The fit is very satisfactory for the whole wavelength interval for all cases. In each comparison we give the parameters of the template spectrum and of the average parameters using only MILES or only Coelho spectra for each star, as presented in Table 6.

use both observational and synthetic libraries. We here used two libraries, one of observed spectra, one of synthetic spectra, as described below.

The MILES library (Sánchez-Blázquez et al. 2006) has 985 stellar spectra with a resolution of $R \sim 2080$ at $5200 \AA$, and a mean signal-to-noise ratio of 150 per pixel for field and open cluster stars, and 50 for globular cluster stars. The atmospheric parameter coverage is (Cenarro et al. 2007; Milone et al. 2011)

$$
\begin{aligned}
& 352.5 \mathrm{~nm}<\lambda<750 \mathrm{~nm} \\
& 2747 \mathrm{~K}<T_{\text {eff }}<36000 \mathrm{~K} \\
& -0.20<\log (g)<5.50 \\
& -2.86<[\mathrm{Fe} / \mathrm{H}]<+1.65 \\
& -0.54<[\mathrm{Mg} / \mathrm{Fe}]<+0.74 \text {. }
\end{aligned}
$$




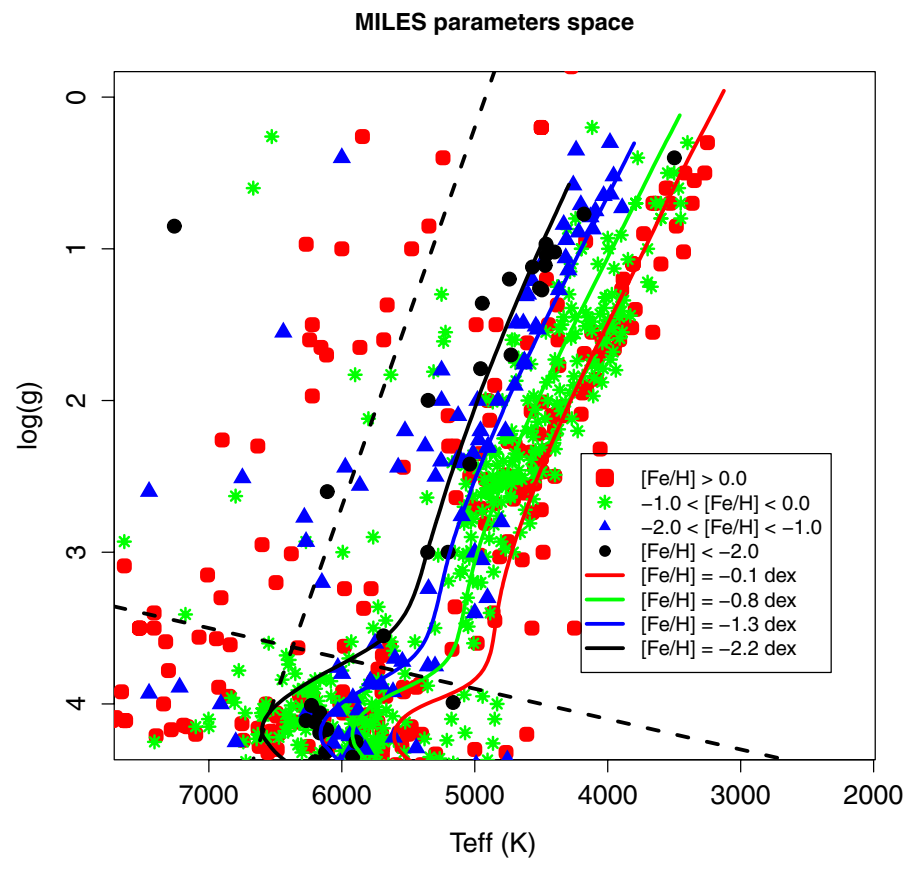

Fig. 6. HR diagram showing the parameter space available from the MILES library. Dartmouth isochrones (Dotter et al. 2008) are overplotted for metallicities close to those of the six analysed globular clusters: $[\mathrm{Fe} / \mathrm{H}]=-0.1,-0.8,-1.3,-2.2 \mathrm{dex}$, with $[\alpha / \mathrm{Fe}]=0.2,0.4,0.4,0.4 \mathrm{dex}$, respectively, and ages $=13 \mathrm{Gyr}$ for all cases. The colours of the dots indicate the metallicity range closer to the isochrones. The dashed black lines are the adopted limit for selecting RGB stars from MILES used as reference for the fits.

The Coelho library (Coelho et al. 2005) has 6367 synthetic stellar spectra ${ }^{4}$ with wavelength steps of $0.02 \AA$ (resolution $R=$ 130000 at $5200 \AA$ ). The atmospheric parameter coverage is

$$
\begin{aligned}
300 \mathrm{~nm} & <\lambda<1800 \mathrm{~nm} \\
3500 \mathrm{~K} & <T_{\mathrm{eff}}<7000 \mathrm{~K} \\
0.0 & <\log (g)<5.0 \\
-2.5 & <[\mathrm{Fe} / \mathrm{H}]<+0.5 \\
0.0 & <[\alpha / \mathrm{Fe}]<+0.4 .
\end{aligned}
$$

The $\alpha$-elements considered in this library are $\mathrm{O}, \mathrm{Mg}, \mathrm{Si}, \mathrm{S}, \mathrm{Ca}$, and Ti.

Given that all cluster stars are located on the red giant branch, as shown in Fig. 1, we selected only stars in this region in the parameter space of the libraries (see Fig. 6) to avoid unphysical results.

\subsubsection{Average results and errors: validation with well-known stars}

We defined different criteria for the MILES and Coelho libraries to average the stellar parameters from reference spectra, as mentioned in Sect. 3.2. For MILES the average results are based on different numbers of templates depending on the sampling, as shown in Fig. 6. For the synthetic library Coelho the sampling is homogeneous, therefore a constant number of templates was adopted. We found that ten templates for Coelho satisfactorily cover the variations in the four stellar parameters $\left(T_{\text {eff }}, \log (g)\right.$, $[\mathrm{Fe} / \mathrm{H}]$, and $[\alpha / \mathrm{Fe}])$. The Coelho library was built by varying all

\footnotetext{
4 We interpolated the original library to produce spectra with $[\alpha / \mathrm{Fe}]=$ $0.1,0.2,0.3$ dex from the provided 0.0 and 0.4 dex spectra.
}

alpha-elements $(\mathrm{O}, \mathrm{Mg}, \mathrm{Si}, \mathrm{S}, \mathrm{Ca}$, and Ti), therefore $[\alpha / \mathrm{Fe}]$ is an average of the enhancement effect of these element abundances.

The criterion to define average results from the MILES library is more complex, as follows: the code provides a list of the closest reference spectra from the library, ranked by the similarity parameter $(S$, as defined in Eqs. (1) and (2)). The final parameters $T_{\text {eff }}, \log (g),[\mathrm{Fe} / \mathrm{H}]$, and $[\mathrm{Mg} / \mathrm{Fe}]$ are the average of the parameters of first $N$ reference stars from the ETOILE output, where $N$ depends on the sampling of the library for each parameter combination. The average is weighted by $1 / S^{2}$ as shown in the equation below for $T_{\text {eff }}$ (the same is valid for the other three parameters):

$T_{\mathrm{eff}}(N)=\frac{\sum_{i=1}^{N} T_{\mathrm{eff}, i} \times \frac{1}{S_{i}^{2}}}{\sum_{i=1}^{N} \frac{1}{S_{i}^{2}}}$.

The errors are defined as the average of the squared residuals, weighted by $1 / S^{2}$, as shown in the equation below for $T_{\text {eff }}$ (the same is valid for the other three parameters). For $N=1$, we adopted the same error of $N=2$.

$\sigma_{T_{\text {eff }}(N)}=\sqrt{\frac{\sum_{i=1}^{N}\left(T_{\mathrm{eff}, i}-T_{\mathrm{eff}}\right)^{2} \times \frac{1}{S_{i}^{2}}}{\sum_{i=1}^{N} \frac{1}{S_{i}^{2}}} .}$

To estimate the number of reference stars to be averaged in each case, we proceeded with some tests using 59 spectra of 49 wellknown stars, listed in Table 4 . These stars were selected among red giant stars (same $\log (g)$ and $T_{\text {eff }}$ intervals defined in Fig. 6) presented in the ELODIE library ${ }^{5}$ (Prugniel et al. 2007). Stellar spectra were taken from the ELODIE library and convolved to the FORS2 resolution. Reference atmospheric parameters were averaged from the PASTEL catalogue (Soubiran et al. 2010), and the quality filter was determined by a threshold in the standard deviation: $\sigma_{T_{\text {eff }}}<200 \mathrm{~K}, \sigma_{\log (\mathrm{g})}<0.5$, and $\sigma_{[\mathrm{Fe} / \mathrm{H}]}<0.2$. We calculated the average parameters and respective errors for different $N$ and compared the results with the average values of $T_{\text {eff }}, \log (g),[\mathrm{Fe} / \mathrm{H}]$ from the PASTEL catalogue (Soubiran et al. 2010). We minimized the equation below to find the best $N$ that will give the final parameters and respective errors. This equation considers the distance between the average for a given $N$ and literature average; in this way, all three parameters are minimized simultaneously. Milone et al. (2011) have measured $[\mathrm{Mg} / \mathrm{Fe}]$ for MILES spectra, therefore it is possible to take averages for this parameter as a function of $N$, and use $[\mathrm{Mg} / \mathrm{Fe}]$ for the best $N$ as an estimate of the $\alpha$-enrichment for each star

$R R_{\text {tot }}(N)=\sqrt{R R_{\mathrm{N}}\left(T_{\text {eff }}\right)^{2}+R R_{\mathrm{N}}(\log (g))^{2}+R R_{\mathrm{N}}([\mathrm{Fe} / \mathrm{H}])^{2}}$,

where $R R_{\mathrm{N}}\left(T_{\text {eff }}\right)$ is given by the equation below (the same is valid for the other three parameters):

$R R_{\mathrm{N}}\left(T_{\mathrm{eff}}\right)=\frac{T_{\mathrm{eff}}(N)-T_{\mathrm{eff}}^{(\mathrm{lit})}(N)}{T_{\mathrm{eff}}^{(\mathrm{lit})}(N)}$.

Figure 7 illustrates the finding procedure of $N$ for star HD 122956, showing that ETOILE accurately recover all four

\footnotetext{
5 http://www.obs.u-bordeaux1.fr/m2a/soubiran/elodie_ library.html
} 
HD 122956 (MILES); RRtot $=0.02 ; \quad \mathrm{N}=3$
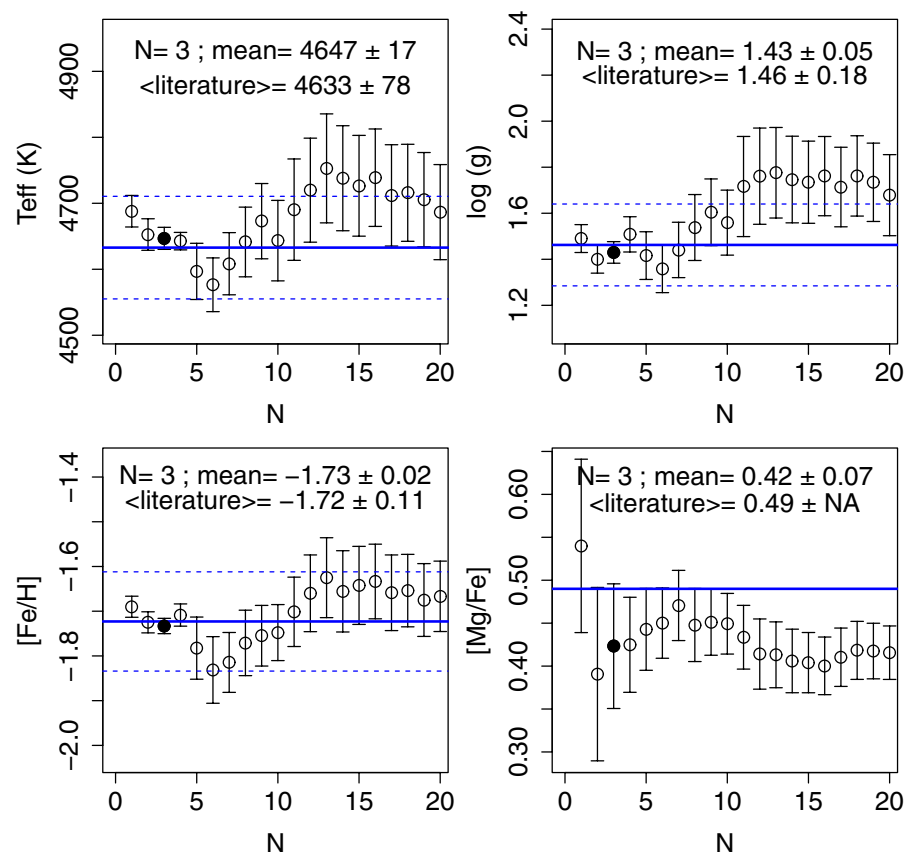

Fig. 7. Finding procedure of $N$ for star HD122956, based on the residual minimization of $T_{\text {eff }}, \log (g)$, and $[\mathrm{Fe} / \mathrm{H}]$ (Eq. (5)). Circles represent the parameter averages of $N$ best-reference stars. The filled black circle indicates the values closest to the references. The blue solid and blue dashed lines are the average from the PASTEL catalogue and the standard deviation (Table 4). For this star, Fulbright (2000) published $[\mathrm{Mg} / \mathrm{Fe}]$, and we compare this also with the averages as a function of $N$. All four parameters for the best $N=3$ are compatible with the literature results.

parameters. The resulting parameters, $R R_{\text {tot }}, N$, and the literature values are presented in Table 4 . Different stars need different numbers $N$ of templates to find the best result. Moreover, the ratio $S(N) / S(1)$ for the best $N$ is roughly constant for all ETOILE template spectra, with an average value of $1.1 \pm 0.1$. The best number $N$ and the respective ratio $S(N) / S(1)$ are related to the library sampling. For example, for a given star with best $N=1$ this means that there is only one reference star with $S(N) / S(1) \lesssim 1.1$. There are two possible explanations: either the target star perfectly matches some reference star, or the library has no other reference spectra similar enough to that star to be considered. For $N=15$, for instance, the library has 15 reference spectra very similar $(S(N) / S(1) \lesssim 1.1)$ to the target spectra, and their parameters must be averaged to obtain the parameters for the target star.

All results are plotted in Fig. 8 and show the good match of ETOILE results and the PASTEL catalogue average for $T_{\text {eff }}$, $\log (g)$, and $[\mathrm{Fe} / \mathrm{H}]$ in the whole range for RGB stars. The behaviour of the derived values of $[\mathrm{Mg} / \mathrm{Fe}]$ vs. $[\mathrm{Fe} / \mathrm{H}]$ is similar to that of field stars (see e.g. Fig. 6 of Alves-Brito et al. 2010).

After these tests we conclude that the code ETOILE together with the MILES library works well for low-resolution spectra of red giant stars in the optical region. Additionally, we define the criterion for considering a reference spectrum similar enough to be suitable for averaging the parameters as $S(N) / S(1) \leq 1.1$.

\section{Results}

The derived $T_{\text {eff }}, \log (g),[\mathrm{Fe} / \mathrm{H}],[\mathrm{Mg} / \mathrm{Fe}]$, and $[\alpha / \mathrm{Fe}]$ are presented in Table 6. We discuss these results as follows: in Sect. 4.1
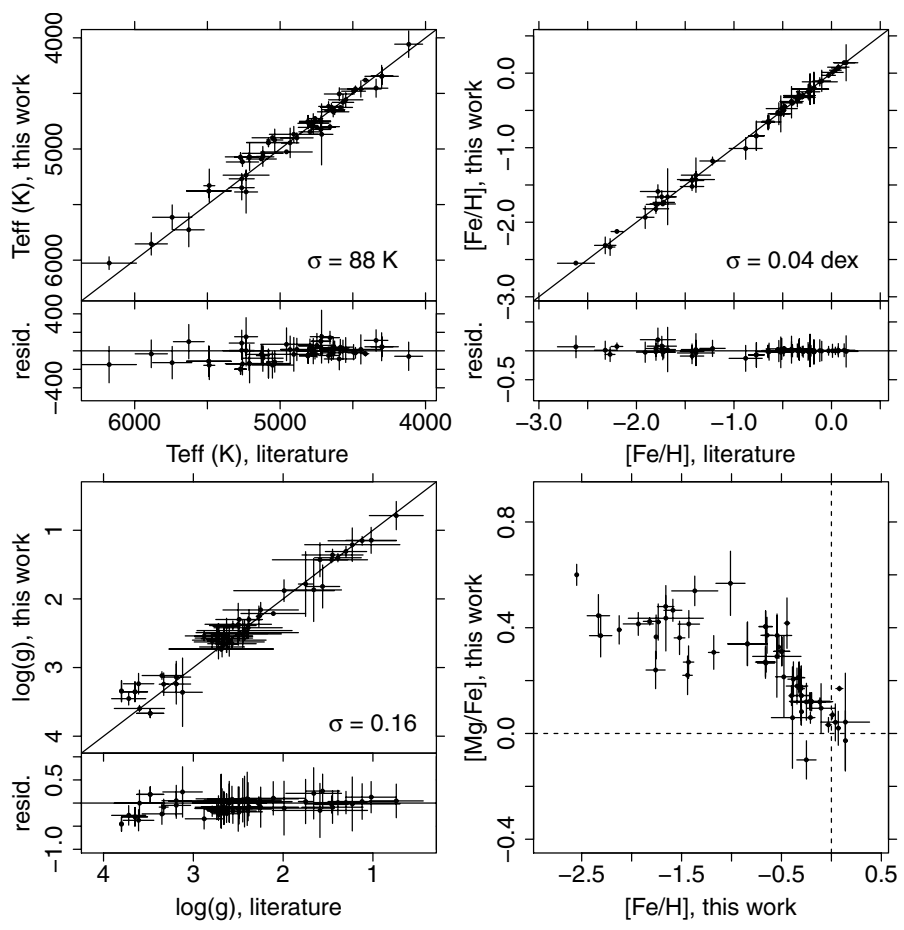

Fig. 8. Comparison of the parameters determined with PASTEL catalogue average values for the well-known stars presented in Table 4. These plots endorse the ETOILE code for atmospheric parameter determination for red giant stars in the optical spectral region. Only stars with $\sigma_{T_{\mathrm{eff}}}<200 \mathrm{~K}, \sigma_{\log (\mathrm{g})}<0.5, \sigma_{[\mathrm{Fe} / \mathrm{H}]}<0.2$ from the PASTEL catalogue were selected as good-quality candidates to validate the method. Below the plots of $T_{\text {eff }}, \log (g)$, and $[\mathrm{Fe} / \mathrm{H}]$ we plot the residuals, the residual dispersion is displayed in the respective plots.

we plot $T_{\text {eff }}$ and $\log (g)$ for stars in each cluster together with isochrones of age and metallicity given in Table 2. Section 4.2 compares $[\mathrm{Fe} / \mathrm{H}]$ with CaT results from Saviane et al. (2012) and Vasquez et al. (in prep.). Subsequently, all checked parameters are used to select member stars for each cluster (Sect. 4.3). Finally, all parameters for member stars are compared individually with a high-resolution analysis, when available in the literature. M 71 and NGC 6558 have three stars in common with the samples studied by Cohen et al. (2001) and Barbuy et al. (2007), and Terzan 8 has four stars in common with Carretta et al. (2014), as described in Sects. 4.4.1-4.4.3, respectively. For NGC 6528, NGC 6553, and NGC 6426 we did not find any star in common with high-resolution spectroscopic studies.

\section{1. $T_{\text {eff }}$ and $\log (g)$ against isochrones}

In high-resolution spectroscopy studies, $T_{\text {eff }}$ is usually estimated from photometry and $\log (g)$ from theoretical equations ${ }^{6}$. These parameters are employed as initial guesses to derive $[\mathrm{Fe} / \mathrm{H}]$, which is applied to redetermine $T_{\text {eff }}$ and $\log (g)$ iteratively, until a convergence of the three parameters is reached. In this work we simultaneously fit all the three parameters (Sect. 3.2), and we checked these parameters as explained below.

Figures 9 to 11 display the results for all stars in the six clusters in a Hertzprung-Russell diagram. The results on $T_{\text {eff }}$ and $\log (g)$ from MILES and Coelho are in good agreement with the isochrones. For reasons explained in Sect. 4.2, we adopted as ${ }_{6} \log (g)=4.44+4 \log \frac{T}{T_{\odot}}$
ple Barbuy et al. (2009). 

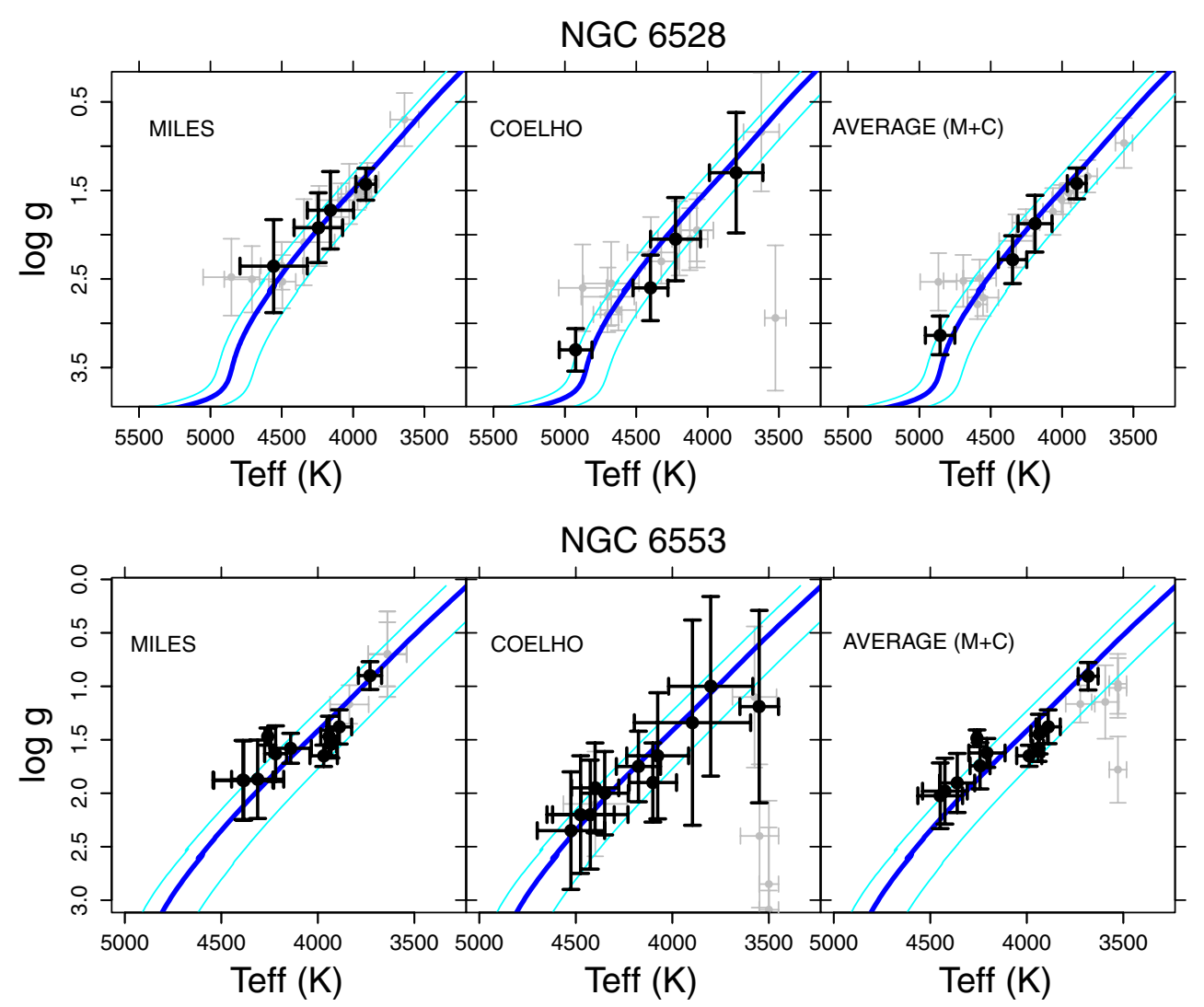

Fig. 9. Comparison of $T_{\text {eff }}$ and $\log (g)$ of stars in each cluster with Dartmouth isochrones (Dotter et al. 2008) for the metal-rich clusters NGC 6528 and NGC 6553. For each cluster we show the results based on the MILES and Coelho libraries and a third panel with the weighted average of the results from both libraries. The parameters of age, $[\mathrm{Fe} / \mathrm{H}]$, and $[\alpha / \mathrm{Fe}]$ for the blue thick isochrones were taken from Table 2 . The cyan thin isochrones have the same age and $[\mathrm{Fe} / \mathrm{H}]$ as the blue ones, but with the limits $[\alpha / \mathrm{Fe}]=-0.2$ dex and +0.8 dex. The black dots represent member stars of each cluster, and grey dots are non-members.

the final results in this work the weighted average of MILES and Coelho results.

\subsection{Comparison of $[\mathrm{Fe} / \mathrm{H}]$ with $\mathrm{CaT}$ results}

When comparing the results from CaT and the optical spectra, it is important to keep in mind that the synthetic spectra in the optical reproduce the metal-rich stars less accurately because of the missing opacity due to millions of very weak lines, which was not taken into account in the calculations; this blanketing effect decreases the continuum in real stars, and the measurable lines are shallower than in the synthetic spectra calculations by Coelho et al. which makes metal-rich stars more similar to synthetic spectra that are slightly more metal-poor. On the other hand, CaT-based abundances also suffer from significant uncertainties. The modelling of the CaT region is affected by contamination by $\mathrm{TiO}$ lines and non-local thermodynamical equilibrium effect. Moreover, measuring a CaT index is very difficult, in particular for more metal-rich and luminous stars with the blanketing effect mentioned above, which complicates defining the continuum for equivalent width (EW) measurements. The conversion of the $\mathrm{EW}$ to $[\mathrm{Fe} / \mathrm{H}]$ has larger uncertainties that might recover even higher $[\mathrm{Fe} / \mathrm{H}]$ for metal-rich stars. Another difficulty of measuring EW for metal-rich $([\mathrm{Fe} / \mathrm{H}] \gtrsim-0.7,47$ Tuc $)$ is choosing the best function to fit the line profile: Gaussian, Gaussian+Lorentzian, or Moffat, while for lower metallicities only a Gaussian function works well. This additional step could introduce uncertainties in $[\mathrm{Fe} / \mathrm{H}]$ from $\mathrm{CaT}$ in metal-rich regime.
Another problem is that the ratio between $[\mathrm{Ca} / \mathrm{H}]$ vs. $[\mathrm{Fe} / \mathrm{H}]$ is not solar, that is, because $\mathrm{Ca}$ is an alpha-element, it is enhanced in old stars, albeit not as enhanced as $\mathrm{O}$ and $\mathrm{Mg}$. A detailed discussion of the CaT metallicities can be found in Saviane et al. (2012). Nevertheless, there are some advantages in comparing our results with CaT: a) all selected stars from photometry were observed both in the near-infrared (CaT, Saviane et al. 2012 and Vasquez et al., in prep.) and in the optical spectral region, which is very useful for comparisons of the whole sample at once; b) the CaT-based metallicities were calibrated with the metallicity scale of Carretta et al. (2009), which makes the CaT metallicities valid at least up to $[\mathrm{Fe} / \mathrm{H}]<-0.43$ (the most metal-rich cluster observed by Carretta et al. 2009, NGC 6441), but metallicities higher than that need to be viewed with caution. Finally, the optical region studied here can easily provide robust values of $[\mathrm{Fe} / \mathrm{H}]$ for each cluster, to compare them with the CaT value, and to converge ultimately to the average $[\mathrm{Fe} / \mathrm{H}]$ for each cluster.

Figure 12 shows the comparisons of the metallicity values presented in Table 6 with those from CaT analysis. The upper left panel compares metallicities using MILES library with $\mathrm{CaT}$ results. The metallicities of the three most metal-rich clusters NGC 6528, NGC 6553, and M 71 match well because of the high number of available MILES templates (see Fig. 6). For NGC 6558 with $[\mathrm{Fe} / \mathrm{H}] \sim-1.0$ the dispersion on the parameters is higher than the CaT results for the three most metalrich clusters, which is explained by the smaller number of stars available in the library with such metallicity. MILES is based on the solar neighbourhood and therefore only shows a few stars 

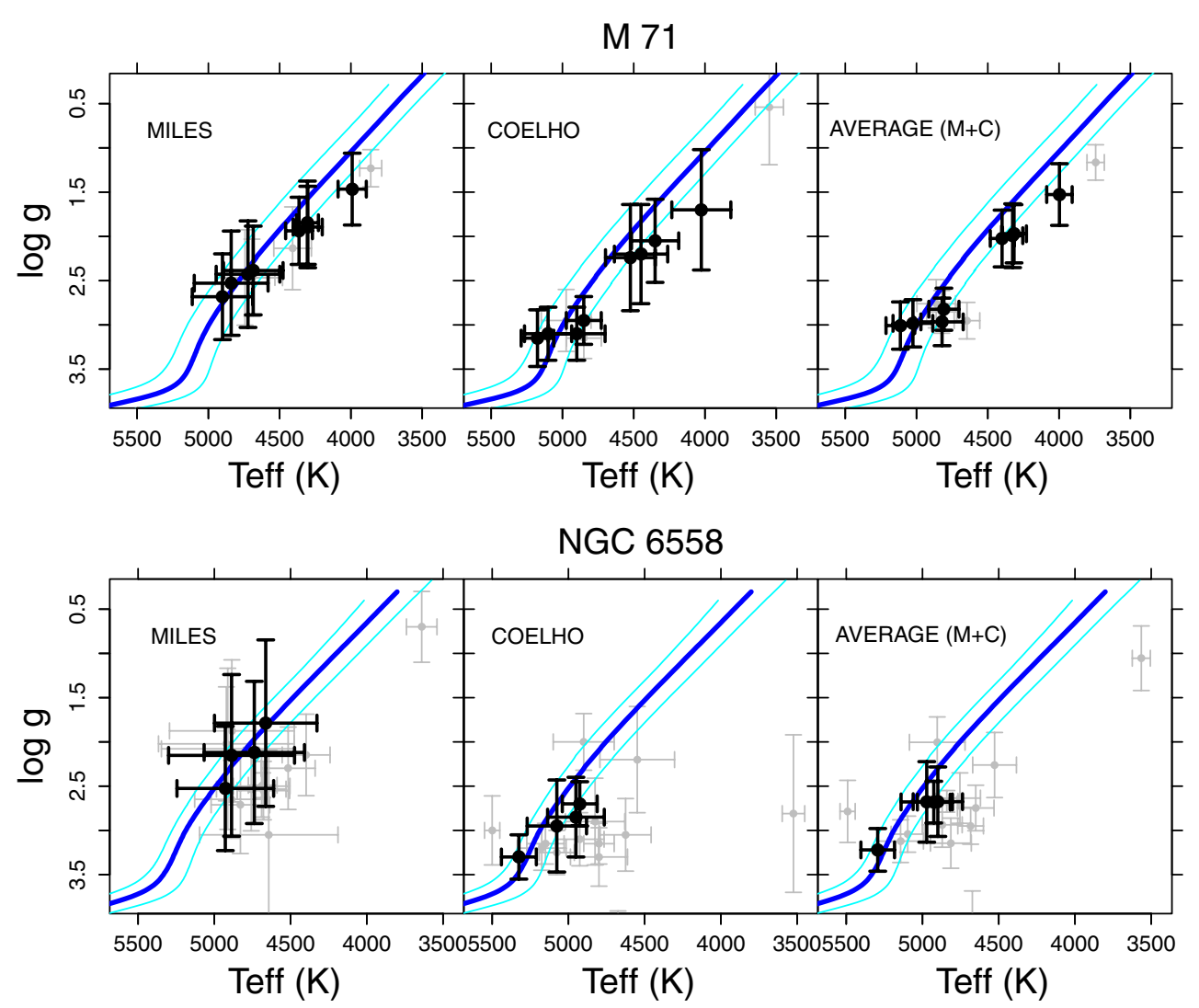

Fig. 10. Same as Fig. 9 for the two intermediate-metallicity clusters M 71 and NGC 6558.

with $[\mathrm{Fe} / \mathrm{H}] \sim-1.0$. For the metal-poor clusters NGC 6426 and Terzan 8 the library sampling is even more sparse, as evident in Fig. 6. In this case, the parameter average from the library takes into account some more metal-rich reference stars, which results in higher values of $[\mathrm{Fe} / \mathrm{H}]$ for NGC 6426 and Terzan 8 stars.

Metallicities using Coelho library are compared with CaT results in the upper right panel of Fig. 12. The synthetic spectra reproduce the metal-rich stars less accurately because of the missing opacity, as mentioned above. Because of this effect, the stars of NGC 6528, NGC 6553, and M71 are more metal-poor than the CaT results. On the other hand, the Coelho library can easily reproduce the stars of the three more metal-poor clusters of this sample, NGC 6558, NGC 6426, and Terzan 8.

To summarize, for the three more metal-rich clusters, MILES results are more accurate, for the other three, the Coelho results are preferable. The bottom right panel of Fig. 12 shows the concatenation of this conclusion, i.e., it displays MILES results for NGC 6528, NGC 6553, and M 71, and Coelho results for NGC 6558, NGC 6426, and Terzan 8. An alternative combination of results from MILES and Coelho is to take the average of the results weighted by their uncertainties. This average combines the best of both libraries and correlates well with CaT results, as shown in the bottom left panel of Fig. 12. Both criteria for combining MILES and Coelho (two bottom panels) agrees well with $\mathrm{CaT}$ results, and we adopted $[\mathrm{Fe} / \mathrm{H}]$ from the average results represented in the bottom left panel.

We adopted as final parameters the mean of MILES and Coelho results, because they are better compatible with the isochrones for $T_{\text {eff }}$ and $\log (g)$, and with the CaT results for metallicities. We recall that CaT-based metallicities were calibrated with the scale of Carretta et al. (2009).

\subsection{Membership selection}

Figure 13 shows NGC 6528 in four panels. The other objects are presented in Figs. 14-18. There is a clear concentration of stars for all clusters around the literature values, and we considered stars with $\Delta v_{\text {helio }}< \pm 2 \sigma$ of the literature value and with $\Delta[\mathrm{Fe} / \mathrm{H}]< \pm 0.3$ dex as members, where $\sigma$ is given in Sect. 3.1 ( similar criteria used by Zoccali et al. 2008, for example). All member stars are close to the isochrones, confirming the membership selection. This extra criterion led to the exclusion of a few more stars from the $v$ helio- $[\mathrm{Fe} / \mathrm{H}]$ selection. Also excluded in some cases are stars cooler than $T_{\text {eff }}<4000 \mathrm{~K}$ that poorly fit the template spectra because of the $\mathrm{TiO}$ bands.

In conclusion, all $T_{\text {eff }}, \log (g)$ and $[\mathrm{Fe} / \mathrm{H}]$ values are found to be located in clearly defined sequences following the isochrones. Our fitting procedure is unaffected by the $[\mathrm{Fe} / \mathrm{H}] / T_{\text {eff }}$ degeneracy, as shown by the comparisons of our results with $[\mathrm{Fe} / \mathrm{H}]$ measurements from the high-resolution analysis available in the literature, that we presented in Sects. 3.2.2 and 4.4.1-4.4.3.

\subsection{Validation with high-resolution spectroscopy}

We found stars in common with previously reported highresolution spectroscopy for three clusters M 71, NGC 6558, and Terzan 8. In Sect. 4.3 we were able to identify ten member stars of M 71, five member stars of NGC 6558, and twelve member stars in Terzan 8 . These are the same as those selected by Saviane et al. (2012) and Vasquez et al. (in prep.). The derived stellar parameters are reported in Table 6 for member and non-member stars. We were able to find detailed analyses in the literature for three member stars in M 71, three in NGC 6558, and four in Terzan 8, as reported below. 


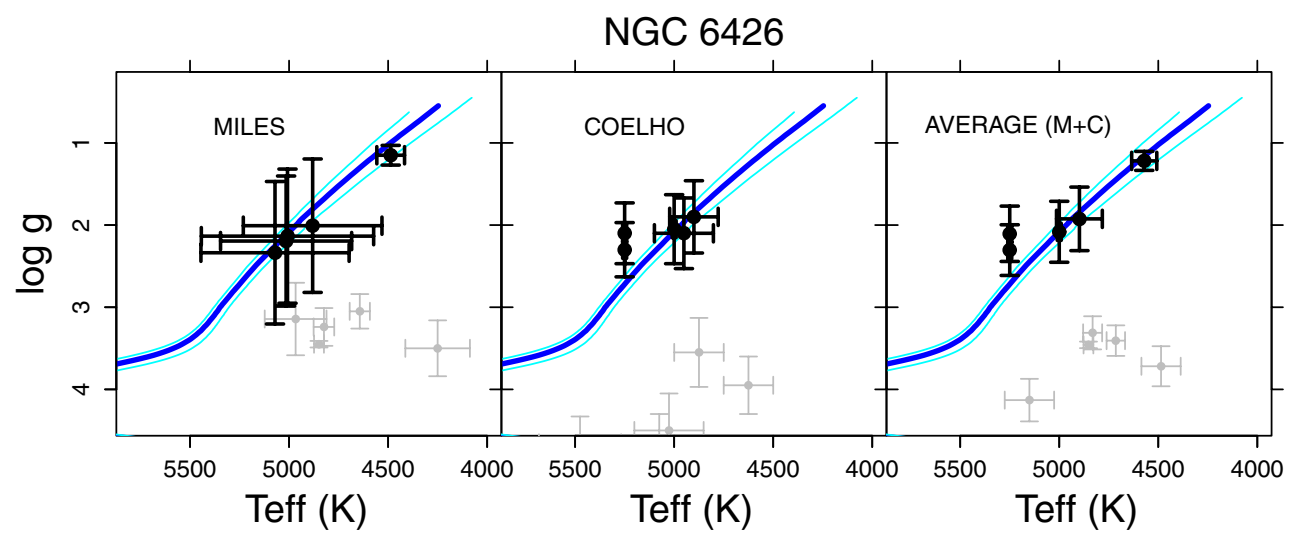

Terzan 8

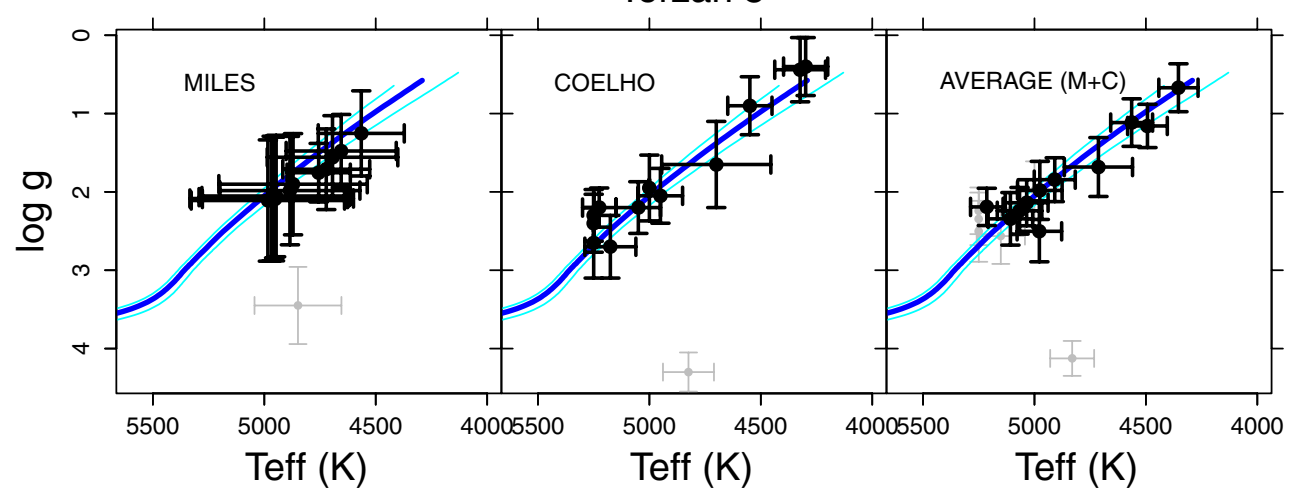

Fig. 11. Same as Fig. 9 for the two metal-poor clusters NGC 6426 and Terzan 8.

Table 5. Final average parameters for member stars in each globular cluster and respective internal errors.

\begin{tabular}{l|cccccc}
\hline \hline Cluster & $\left\langle v_{\text {helio }}\right\rangle\left(\mathrm{km} \mathrm{s}^{-1}\right)$ & $\langle[\mathrm{Fe} / \mathrm{H}]\rangle^{a}$ & $\langle[\mathrm{Fe} / \mathrm{H}]\rangle^{b}$ & $\langle[\mathrm{Fe} / \mathrm{H}]\rangle^{\text {avg }}$ & $\langle[\mathrm{Mg} / \mathrm{Fe}]\rangle^{a}$ & $\langle[\alpha / \mathrm{Fe}]\rangle^{b}$ \\
\hline NGC 6528 & $185 \pm 10$ & $-0.07 \pm 0.10$ & $-0.18 \pm 0.08$ & $-0.13 \pm 0.05$ & $0.05 \pm 0.09$ & $0.26 \pm 0.05$ \\
NGC 6553 & $6 \pm 8$ & $-0.125 \pm 0.009$ & $-0.55 \pm 0.07$ & $-0.133 \pm 0.017$ & $0.107 \pm 0.009$ & $0.302 \pm 0.025$ \\
M 71 & $-42 \pm 18$ & $-0.48 \pm 0.08$ & $-0.77 \pm 0.08$ & $-0.63 \pm 0.15$ & $0.25 \pm 0.07$ & $0.293 \pm 0.032$ \\
NGC 6558 & $-210 \pm 16$ & $-0.88 \pm 0.20$ & $-1.02 \pm 0.05$ & $-1.012 \pm 0.013$ & $0.26 \pm 0.06$ & $0.23 \pm 0.06$ \\
NGC 6426 & $-242 \pm 11$ & $-2.03 \pm 0.11$ & $-2.46 \pm 0.05$ & $-2.39 \pm 0.11$ & $0.38 \pm 0.06$ & $0.24 \pm 0.05$ \\
Terzan 8 & $135 \pm 19$ & $-1.76 \pm 0.07$ & $-2.18 \pm 0.05$ & $-2.06 \pm 0.17$ & $0.41 \pm 0.04$ & $0.21 \pm 0.04$ \\
\hline
\end{tabular}

Notes. ${ }^{(a)}$ MILES library; ${ }^{(b)}$ Coelho library; ${ }^{(a v g)}$ Average of MILES and Coelho results.

\subsubsection{71}

Cohen et al. (2001) observed 25 member red giant stars of M 71 using HIRES at Keck $(R \sim 34000)$, and derived their $T_{\text {eff }}$ and $\log (g)$. In two subsequent papers, they derived [Fe/H] (Ramírez et al. 2001) and [Mg/Fe] (Ramírez \& Cohen 2002) for them. We have three stars in common that are presented in Table 7. Temperature and gravity values are compatible within 0.5 to $2 \sigma$, $[\mathrm{Fe} / \mathrm{H}]$ and $[\mathrm{Mg} / \mathrm{Fe}]$ are compatible within 0.1 to $1.5 \sigma$.

\subsubsection{NGC 6558}

Barbuy et al. (2007) observed six RGB stars using the highresolution $(R \sim 22000)$ spectrograph FLAMES+GIRAFFE at $\mathrm{VLT} / \mathrm{ESO}$, and derived $T_{\mathrm{eff}}, \log (g),[\mathrm{Fe} / \mathrm{H}]$, and $[\mathrm{Mg} / \mathrm{Fe}]$ for each of them. We have three stars in common with their sample: 6 , 8,9 , corresponding to their identification as B11, F42, F97, respectively (see Table 8). For stars 6 and 9, full spectrum fitting recovers all parameters within $1 \sigma$. Star 8 is a more complicated case because it is a very cool star $\left(T_{\text {eff }}<4000 \mathrm{~K}\right)$ with strong
Table 7. Final atmospheric parameters for the three stars of M 71 in common with Cohen et al. (2001), and their determinations for the respective parameters.

\begin{tabular}{lllll}
\hline \hline Star & $\begin{array}{l}T_{\text {eff }}(\mathrm{K}) \\
T_{\text {eff }}-\mathrm{C01}(\mathrm{K})\end{array}$ & $\begin{array}{l}\log (g) \\
\log (g)-\mathrm{C} 01\end{array}$ & $\begin{array}{l}{[\mathrm{Fe} / \mathrm{H}]} \\
{[\mathrm{Fe} / \mathrm{H}]-\mathrm{C} 01}\end{array}$ & $\begin{array}{l}{[\mathrm{Mg} / \mathrm{Fe}]} \\
{[\mathrm{Mg} / \mathrm{Fe}]-\mathrm{C} 01}\end{array}$ \\
\hline M71_7 & $3997 \pm 89$ & $1.53 \pm 0.35$ & $-0.58 \pm 0.17$ & $0.15 \pm 0.18$ \\
1-45 & 3950 & 0.9 & $-0.60 \pm 0.03$ & $0.43 \pm 0.09$ \\
M71_9 & $4316 \pm 87$ & $1.97 \pm 0.33$ & $-0.76 \pm 0.17$ & $0.27 \pm 0.21$ \\
1-64 & 4200 & 1.35 & $-0.61 \pm 0.03$ & $0.43 \pm 0.09$ \\
M71_13 & $4808 \pm 106$ & $2.82 \pm 0.24$ & $-0.63 \pm 0.18$ & $0.23 \pm 0.20$ \\
G53476_4543 & 4900 & 2.65 & $-0.61 \pm 0.03$ & $0.36 \pm 0.06$ \\
\hline
\end{tabular}

molecular bands of TiO. They strongly change the continuum, which is not fitted perfectly. In fact, the derived parameters for this star led us to exclude it. Although the temperature agrees with the temperature reported in Barbuy et al. (2007), the gravity is much lower than their results. 

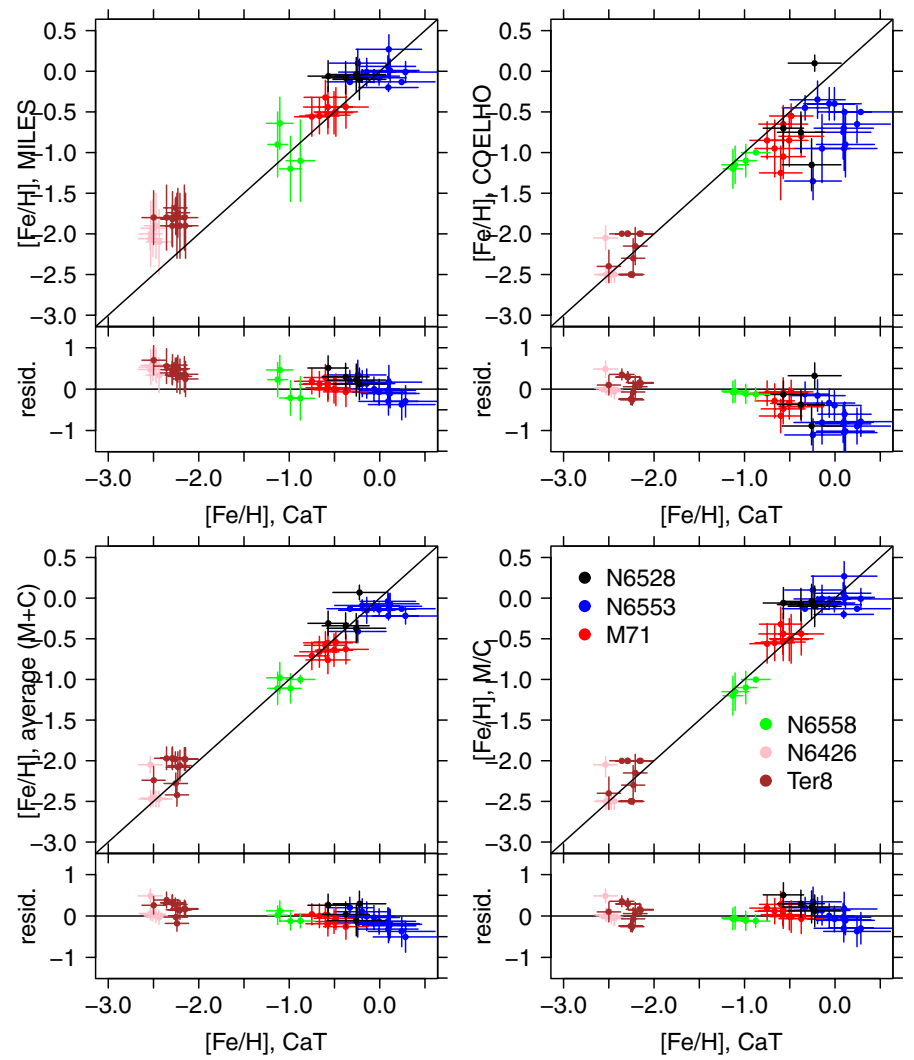

Fig. 12. Comparison of $[\mathrm{Fe} / \mathrm{H}]$ from this work (mean of MILES and Coelho, see Table 6) with those from equivalent widths of Ca II triplet for the same stars with the same instrument by Saviane et al. (2012) and Vasquez et al. (in prep). The upper panels compare CaT metallicities with those obtained with the MILES and Coelho libraries. The bottom panels are two types of combination of the results: the average of each star on the left, and assuming MILES results for more metal-rich and Coelho results for more metal-poor stars on the right. Below the plots we plot the residuals.

Table 8. Final atmospheric parameters for the three stars of NGC 6558 in common with Barbuy et al. (2007), and their determinations for the respective parameters.

\begin{tabular}{lllll}
\hline \hline Star & $\begin{array}{l}T_{\text {eff }}(\mathrm{K}) \\
T_{\text {eff }}-\mathrm{B} 07(\mathrm{~K})\end{array}$ & $\begin{array}{l}\log (g) \\
\log (g)-\mathrm{B} 07\end{array}$ & $\begin{array}{l}{[\mathrm{Fe} / \mathrm{H}]} \\
{[\mathrm{Fe} / \mathrm{H}]-\mathrm{B} 07}\end{array}$ & $\begin{array}{l}{[\mathrm{Mg} / \mathrm{Fe}]} \\
{[\mathrm{Mg} / \mathrm{Fe}]-\mathrm{B} 07}\end{array}$ \\
\hline $6558 \_6$ & $4899 \pm 162$ & $2.68 \pm 0.39$ & $-1.11 \pm 0.20$ & $0.22 \pm 0.07$ \\
B11 & 4650 & 2.2 & -1.04 & 0.20 \\
$6558 \_8$ & $3565 \pm 59$ & $1.05 \pm 0.36$ & $-0.16 \pm 0.06$ & $0.23 \pm 0.00$ \\
F42 & 3800 & 0.5 & -1.01 & 0.30 \\
6558_9 & $4972 \pm 168$ & $2.68 \pm 0.46$ & $-1.11 \pm 0.18$ & $0.41 \pm 0.16$ \\
F97 & 4820 & 2.3 & -0.97 & 0.23 \\
\hline
\end{tabular}

These results show that the full spectrum fitting method is reliable, consistent among all libraries, and presents reasonable errors for RGB stars hotter than $\sim 4000 \mathrm{~K}$. Stars cooler than that must be analysed with a better suited reference library that needs to cointain a sufficient number of cool stars at all metallicities.

\subsubsection{Terzan 8}

Carretta et al. (2014) observed six stars with UVES at VLT/ESO $(R \sim 45000)$ and 14 with GIRAFFE at VLT/ESO $(R \sim$ 22 500-24200), four stars of which are in common with our
NGC 6528
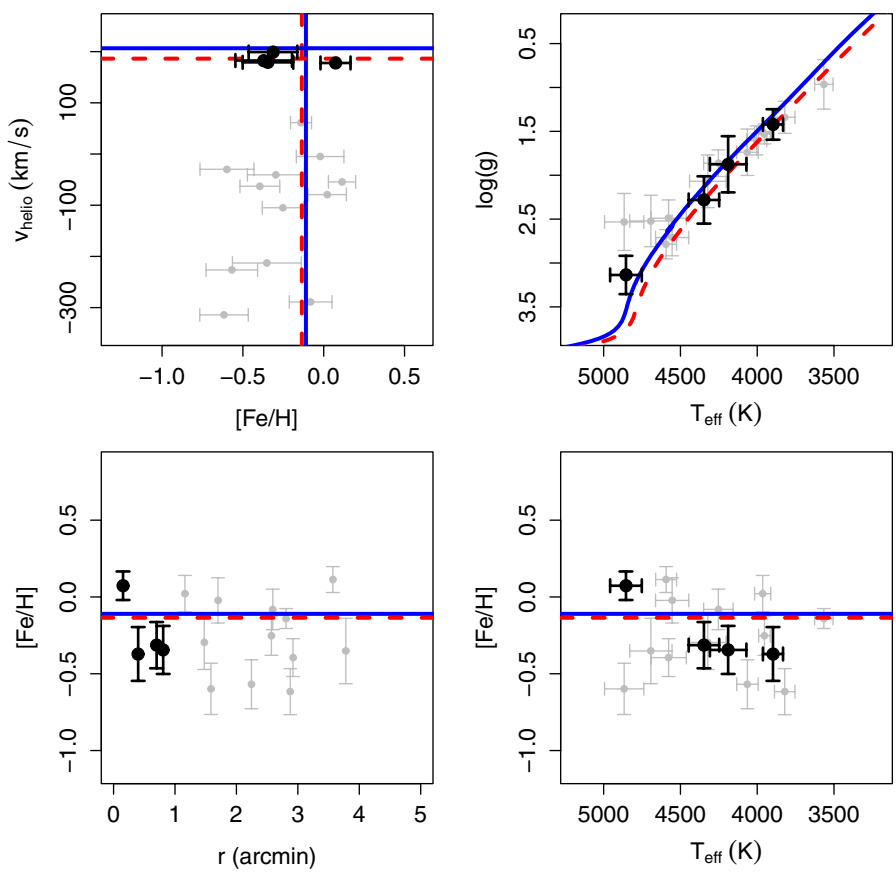

Fig. 13. Step-by-step of the member star selection for NGC 6528. The black dots are selected member stars, grey dots are non-members, and green circles show stars considered as cluster member by Saviane et al. (2012), but non-members in the present work. The blue solid lines are drawn based on values of Table 2, which were also applied to the ischrones from Dotter et al. (2008). For isochrones we use age, $[\mathrm{Fe} / \mathrm{H}]$ and $[\alpha / \mathrm{Fe}]$ information. The red dashed lines refer to the weighted average of the member star parameters.

Table 9. Final atmospheric parameters for the four stars of Terzan 8 in common with Carretta et al. (2014), together with their determinations for the respective parameters.

\begin{tabular}{lllll}
\hline \hline Star & $T_{\text {eff }}(\mathrm{K})$ & $\log (g)$ & {$[\mathrm{Fe} / \mathrm{H}]$} & {$[\mathrm{Mg} / \mathrm{Fe}]$} \\
& $T_{\text {eff }}-\mathrm{C} 14(\mathrm{~K})$ & $\log (g)-\mathrm{C} 14$ & {$[\mathrm{Fe} / \mathrm{H}]-\mathrm{C} 14$} & {$[\mathrm{Mg} / \mathrm{Fe}]-\mathrm{C} 14$} \\
\hline Ter8_1 & $5067 \pm 314$ & $2.24 \pm 0.30$ & $-1.97 \pm 0.14$ & $0.40 \pm 0.13$ \\
2913 & 4628 & 1.49 & $-2.52 \pm 0.07$ & 0.58 \\
Ter8_4 & $4354 \pm 88$ & $0.67 \pm 0.31$ & $-2.28 \pm 0.13$ & $0.40 \pm 0.14$ \\
2357 & 4188 & 0.66 & $-2.29 \pm 0.10$ & $0.48 \pm 0.14$ \\
Ter8_8 & $5151 \pm 108$ & $2.56 \pm 0.36$ & $-2.06 \pm 0.19$ & $0.40 \pm 0.18$ \\
2124 & 4730 & 1.67 & $-2.28 \pm 0.26$ & 0.56 \\
Ter8_9 & $4564 \pm 94$ & $1.12 \pm 0.30$ & $-2.24 \pm 0.17$ & $0.42 \pm 0.13$ \\
1658 & 4264 & 0.80 & $-2.40 \pm 0.07$ & $0.51 \pm 0.02$ \\
\hline
\end{tabular}

sample observed with FORS2 at VLT/ESO. Their parameters for these stars are presented in Table 9. Temperature and gravity are compatible to within 1 to $3 \sigma$, except for $T_{\text {eff }}$ of star Ter8_8, which is in the limit of $3.9 \sigma$ of distance. For $[\mathrm{Fe} / \mathrm{H}]$ all stars have values compatible with those reported in Carretta et al. (2014) within $1 \sigma$, except for star Ter8_1, which is in the limit of $3.9 \sigma$ of distance. $[\mathrm{Mg} / \mathrm{Fe}]$ is compatible within $1 \sigma$.

Differently from M71 and NGC 6558, the comparison between our results and those of Carretta et al. (2014) for Terzan 8 give all three parameters $T_{\text {eff }}, \log (g)$, and $[\mathrm{Fe} / \mathrm{H}]$ as systematically larger. For this reason we inspected the $T_{\text {eff }}-\log (g)$ diagram of both sets of data and compared it with the Dartmouth (Dotter et al. 2008), PARSEC (Bressan et al. 2012) and BASTI (Pietrinferni et al. 2004) isochrones, as shown in Fig. 19. The 
B. Dias et al.: FORS2/VLT survey of Milky Way globular clusters. I.

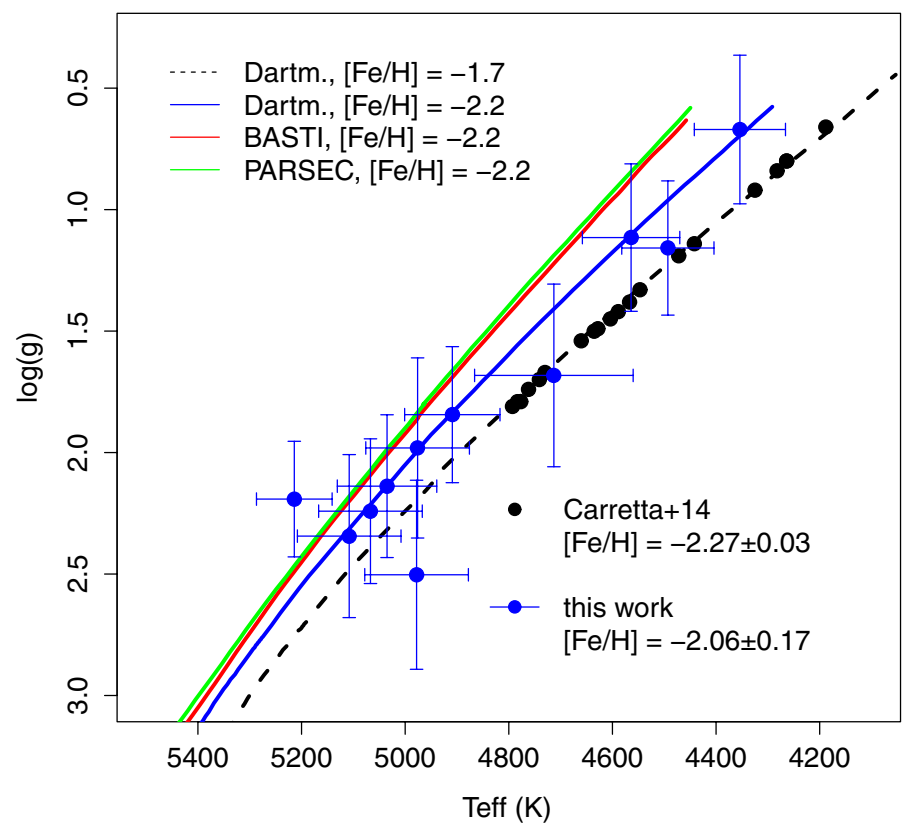

Fig. 19. Diagram $\log (g)$ vs. $T_{\text {eff }}$ for Terzan 8 showing our results (blue points with error bars) and those of Carretta et al. (2014, black filled circles), overplotted with Dartmouth, PARSEC, and BASTI isochrones for ages = $13 \mathrm{Gyr}$, and metallicities indicated in the figure.

Carretta et al. (2014) results are compatible with an isochrone of $[\mathrm{Fe} / \mathrm{H}]=-1.7,[\alpha / \mathrm{Fe}]=+0.4$ and $13 \mathrm{Gyr}$, whereas our results fit better with an isochrone $[\mathrm{Fe} / \mathrm{H}]=-2.2,[\alpha / \mathrm{Fe}]=+0.4$ and 13 Gyr. Except for star Terzan8_4, which shows very similar gravities, the gravities are different for the other stars. Given that the results are consistent with isochrones, we suggest that for a high-resolution analysis of metal-poor stars, the effect of over-ionization at low temperature atmospheres may have led to lower gravities.

\section{Discussion}

The results for individual stars in each cluster (Table 6) and the average results (Table 5) are discussed below and are compared with literature results. Figure 20 displays the comparison of our $[\mathrm{Fe} / \mathrm{H}]$ results for each cluster with reference values, showing good agreement for the whole range of metallicities from $[\mathrm{Fe} / \mathrm{H}]=-2.5$ to solar. Figure 21 gives the comparison of the average results of $[\mathrm{Fe} / \mathrm{H}]$ and $[\mathrm{Mg} / \mathrm{Fe}]$ with abundances for field stars of the different Galactic components: bulge, thin and thick disc, and inner and outer halo. We discuss case by case below.

\subsection{Metal-rich clusters NGC 6528 and NGC 6553}

The clusters NGC 6528 and NGC 6553 have similar CMDs (Ortolani et al. 1995), as shown in Fig. 1. Their metallicities and element abundance ratios are also similar for most elements, as reported in Table 10. As listed in Table 2, they have reddening $E(B-V)=0.54 \mathrm{mag}$ and $0.63 \mathrm{mag},[\mathrm{Fe} / \mathrm{H}]=-0.11$ and $-0.18,[\mathrm{Mg} / \mathrm{Fe}]=0.24$ and 0.26 , respectively. They are located in the Milky Way bulge, at a distance of $0.6 \mathrm{kpc}$ and $2.2 \mathrm{kpc}$ from the Galactic centre and in the opposite southern legs of the X-shaped bulge (see Fig. 3 of Saito et al. 2011). Figure 9 shows that ETOILE recovers parameters for member stars coherent with the simple stellar population represented by the isochrones. Although all the results are compatible between the libraries,

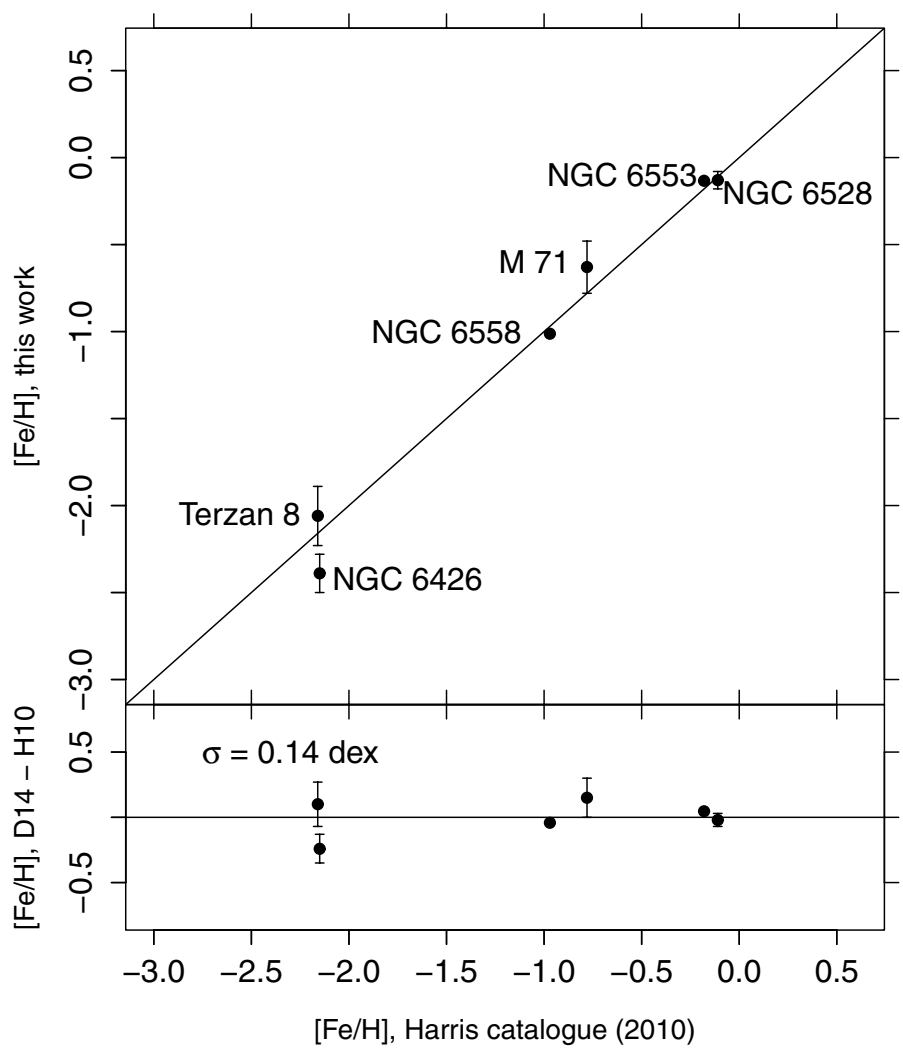

Fig. 20. Comparison of $[\mathrm{Fe} / \mathrm{H}]$ from this work (Table 5) with those from literature, as revised in Table 2. The error bars are the weighted average of the $[\mathrm{Fe} / \mathrm{H}]$ of member stars in each cluster, as presented in Table 6 . For NGC 6553 and NGC 6558 the error bars are not visible because they are too small (see Table 5). In the lower panel we show the residuals.

error bars for MILES results are lower than from Coelho results. This synthetic library, on the other hand, gives better alphaenhancement values that are compatible with the average values from high-resolution studies $([\alpha / \mathrm{Fe}]=0.26 \pm 0.05$ and $0.302 \pm 0.025$, respectively, from Table 5). In particular, these values are compatible with NGC 6553 (Alves-Brito et al. 2006; Cohen et al. 1999), while for NGC 6528 the alpha-enhancement is lower (Zoccali et al. 2004). In this respect, MILES cannot give an alpha-enhancement, since their metal-rich stars are basically solar neighbourhood stars that have no alpha-enhancement for metal-rich stars (see Fig. 2 in Milone et al. 2011). The results from MILES are $[\mathrm{Mg} / \mathrm{Fe}]=0.05 \pm 0.09$ and $0.107 \pm 0.009$. This is a particularity of the bulge, where stars are metalrich and old. As mentioned above, Zoccali et al. (2004) found $[\mathrm{Mg} / \mathrm{Fe}]=+0.07$ from high-resolution spectroscopy of three stars of NGC 6528, which is compatible with MILES and not with Coelho. For N6553, Cohen et al. (1999) also found $[\mathrm{Mg} / \mathrm{Fe}]=+0.41$ from high-resolution spectroscopy of five stars, which is closer to Coelho results.

Figure 13 compares the final results (average of MILES and Coelho results) with isochrones with literature parameters (same as Fig. 9) and an additional isochrone considering the parameters derived from this work (Table 5). The isochrones consider $[\alpha / \mathrm{Fe}]$ from Coelho results as discussed above. We derived $[\mathrm{Fe} / \mathrm{H}]=$ $-0.13 \pm 0.06$ and $-0.133 \pm 0.009$ for NGC 6528 and NGC 6553, respectively, in agreement with high spectral resolution analyses of Carretta et al. (2001) and Alves-Brito et al. (2006). 
Table 10. Literature abundances for NGC 6528 and NGC 6553.

\begin{tabular}{lccccccccccc}
\hline \hline$[\mathrm{Fe} / \mathrm{H}]$ & {$[\mathrm{O} / \mathrm{Fe}]$} & {$[\mathrm{Mg} / \mathrm{Fe}]$} & {$[\mathrm{Si} / \mathrm{Fe}]$} & {$[\mathrm{Ca} / \mathrm{Fe}]$} & {$[\mathrm{Ti} / \mathrm{Fe}]$} & {$[\mathrm{Na} / \mathrm{Fe}]$} & {$[\mathrm{Eu} / \mathrm{Fe}]$} & {$[\mathrm{Ba} / \mathrm{Fe}]$} & {$[\mathrm{Mn} / \mathrm{Fe}]$} & {$[\mathrm{Sc} / \mathrm{Fe}]$} & $\mathrm{Ref}$. \\
\hline NGC 6528 & & & & & & & & & & & \\
\hline+0.07 & +0.07 & +0.14 & +0.36 & +0.23 & +0.03 & +0.40 & - & +0.14 & -0.37 & -0.05 & $(1)$ \\
-0.11 & +0.10 & +0.05 & +0.05 & -0.40 & -0.25 & +0.60 & - & - & - & - & $(2)$ \\
\hline NGC 6553 & & & & & & & & & & \\
\hline-0.16 & +0.50 & +0.41 & +0.14 & +0.26 & +0.19 & - & - & - & - & -0.12 & $(3)$ \\
-0.20 & +0.20 & - & - & - & - & - & - & - & - & - & $(4)$ \\
-0.20 & - & +0.28 & +0.21 & +0.05 & -0.01 & +0.16 & +0.10 & -0.28 & - & - & $(5)$ \\
\hline
\end{tabular}

Notes. ${ }^{(1)}$ Carretta et al. (2001); ${ }^{(2)}$ Zoccali et al. (2004); (3) Cohen et al. (1999); ${ }^{(4)}$ Meléndez et al. (2003); (5) Alves-Brito et al. (2006).

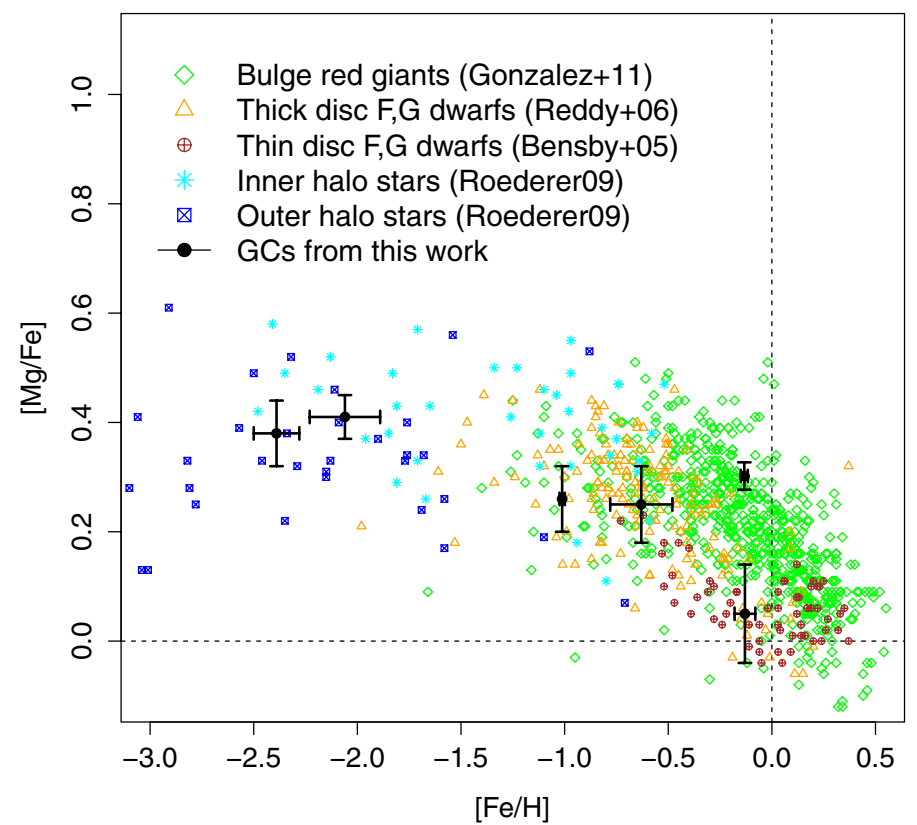

Fig. 21. Chemical evolution of the Milky Way based on bulge field stars (Gonzalez et al. 2011), thin disc (Bensby et al. 2005), thick disc (Reddy et al. 2006), and inner and outer halo stars (Roederer 2009). Our results for globular clusters are overplotted. For NGC 6553 the point corresponds to $[\alpha / \mathrm{Fe}]$ instead of $[\mathrm{Mg} / \mathrm{Fe}]$, as discussed in Sect. 5.1

\subsection{Moderately metal-rich clusters M71 and NGC 6558}

According to Harris (1996, 2010 edition), M 71 (or NGC 6838) is located $6.7 \mathrm{kpc}$ away from the Galactic centre, with a perpendicular distance to the Galactic plane of only $0.3 \mathrm{kpc}$ towards the South Galactic Pole, which means that this globular cluster is located in the Milky Way disc. Because of this its reddening is high, although not as high as for most bulge clusters, with $E(B-V)=0.25$. NGC 6558 is located only $1.0 \mathrm{kpc}$ from the Galactic centre, in particular between the two southern legs of the X-shaped bulge (see Fig. 3 of Saito et al. 2011). It has a reddening of $E(B-V)=0.44$. Although they are located in different components of the Milky Way, these clusters share similar metallicities $[\mathrm{Fe} / \mathrm{H}] \sim-0.8$, and $[\mathrm{Fe} / \mathrm{H}] \sim-1.0$.

Figure 1 shows a red horizontal branch (HB) for M71 and a blue HB for NGC 6558, and this difference is not due to metallicity (Lee et al. 1994). It is true that a few other parameters can change the $\mathrm{HB}$ morphology at a fixed metallicity, as discussed by Catelan et al. (2001), for instance, but in this case the age is probably playing the main role. Literature ages are 11 Gyr (VandenBerg et al. 2013) and 14 Gyr old (Barbuy et al. 2007). M 71 is younger and moderately metal-rich, therefore a red horizontal branch is expected. In particular, we derived a slightly higher metallicity for this cluster than is reported in the literature, $[\mathrm{Fe} / \mathrm{H}]=-0.63 \pm 0.06$. NGC 6558 has a high metallicity for such a blue horizontal branch, and Barbuy et al. (2007) argued that if this is interpreted as a pure age factor, this cluster is one of the oldest objects in the Milky Way. We derived $[\mathrm{Fe} / \mathrm{H}]=-1.01 \pm 0.04$, compatible with their findings $([\mathrm{Fe} / \mathrm{H}]=-0.97 \pm 0.15)$, and more metal-rich than the value of $[\mathrm{Fe} / \mathrm{H}]=-1.32$ reported in Harris $(1996,2010$ edition). Saviane et al. (2012) also found $[\mathrm{Fe} / \mathrm{H}]=-1.03 \pm 0.14$ for NGC 6558 from their Ca II triplet spectroscopy; their error was dominated by the external calibration uncertainty.

A comparison of the error bars of $T_{\text {eff }}$ and $\log (g)$ in Fig. 10 between the two libraries shows that for M 71 they are of the same order, but for NGC 6558 the MILES results present larger error bars. The main reason for this is that MILES library is based on solar neighbouhood stars, and only a few of them have metallicities $[\mathrm{Fe} / \mathrm{H}] \sim-1.0$ (see Fig. 2 of Sánchez-Blázquez et al. 2006). Synthetic libraries such as that of Coelho et al. (2005) have spectra for any combination of atmospheric parameters evenly distributed in the parameter space. Therefore the Coelho library is more suitable for the analysis of these moderately metal-rich clusters.

For this range of metallicities and for expected values of $[\mathrm{Mg} / \mathrm{Fe}]$ from the literature $(0.19$ and 0.24$)$, the MILES and Coelho results are compatible, as shown in Table 5 $[\mathrm{Mg} / \mathrm{Fe}]=0.25 \pm 0.07$ and $0.26 \pm 0.06,[\alpha / \mathrm{Fe}]=0.293 \pm 0.032$ and $0.23 \pm 0.06$. Ramírez \& Cohen (2002) give an average $[\mathrm{Mg} / \mathrm{Fe}]=+0.37$ from 24 stars observed with high-resolution in M71, which is compatible with our findings. Few stars in MILES have these metallicities, but they are sampled well enough to determine $[\mathrm{Fe} / \mathrm{H}]$ and $[\mathrm{Mg} / \mathrm{Fe}]$ for Milky Way stars. Coelho spectra have $[\alpha / \mathrm{Fe}]$ varying from 0.0 to 0.4 , which is also enough for these objects.

\subsection{Metal-poor clusters NGC 6426 and Terzan 8}

The clusters NGC 6426 and Terzan 8 present similar CMDs with a same literature age and metallicity of 13 Gyr (Dotter et al. 2011; VandenBerg et al. 2013) and $[\mathrm{Fe} / \mathrm{H}]=-2.15$ (Harris 1996, 2010 edition). NGC 6426 is located in the northern halo of the Milky Way, $14.4 \mathrm{kpc}$ away from the Galactic centre and $5.8 \mathrm{kpc}$ above the Galactic plane. Its height is much greater than the height scale of the thick disc $(0.75 \mathrm{kpc}$, de Jong et al. 2010), but it has a considerable reddening of $E(B-V)=0.36$ at a galactic latitude of $b=16.23^{\circ}$. The best CMD available for this cluster was observed with the ACS imager onboard the Hubble Space Telescope by Dotter et al. (2011), who derived an age of $13.0 \pm 1.5$ Gyr from isochrone fitting. Our pre-image photometry 
based on observations with the Very Large Telescope of ESO produced a rather well-defined CMD for this cluster, which is compatible with a $13 \mathrm{Gyr}$ isochrone (see Fig. 1).

Terzan 8 is located in the southern halo of the Milky Way, $19.4 \mathrm{kpc}$ away from the Galactic centre and $10.9 \mathrm{kpc}$ below the Galactic plane. It has the lowest reddening of all clusters analysed in this work, $E(B-V)=0.12$. This is one of the four Milky Way globular clusters believed to be captured from the Sagittarius dwarf galaxy (Ibata et al. 1994), the other three being M 54, Terzan 7, and Arp 2 (Da Costa \& Armandroff 1995). Carretta et al. (2014) did not find strong evidence for a $\mathrm{Na}-\mathrm{O}$ anticorrelation that is typical for globular clusters, which may indicate that these clusters may have simple stellar populations.

Atmospheric parameters derived in this work for both clusters agree well with literature values when compared with the isochrones in Fig. 11. For the moderately metal-rich clusters, MILES results present larger error bars than Coelho results for $T_{\text {eff }}$ and $\log (g)$; the reason is the same as mentioned above, that is, the sampling of the MILES library is poorer for this metallicity range (see Fig. 2 of Sánchez-Blázquez et al. 2006). The derived metallicities for these clusters are $[\mathrm{Fe} / \mathrm{H}]=-2.39 \pm 0.04$ and $-2.06 \pm 0.04$. For NGC 6426 our determination is 0.24 more metal-poor than given in the catalogue of Harris $([\mathrm{Fe} / \mathrm{H}]=$ $-2.15)$. However, the only derivation of the metallicity for this cluster was done by Zinn \& West (1984) based on integrated light $([\mathrm{Fe} / \mathrm{H}]=-2.20 \pm 0.17)$, which is compatible with our findings. The value from the Harris catalogue was obtained by applying the metallicity scale of Carretta et al. (2009). We present for the first time a direct measurement of metallicity of individual red giant stars and find a more metal-poor value than previously attributed to this cluster. Terzan 8 is compatible with the Harris (2010 edition) catalogue of $[\mathrm{Fe} / \mathrm{H}]=-2.16$. Three metallicities have been reported for this cluster: Mottini et al. (2008) and Carretta et al. (2014) studied the metallicities based on high-resolution spectra of individual stars and average metallicity $[\mathrm{Fe} / \mathrm{H}]=-2.35 \pm 0.04$ and $-2.27 \pm 0.08$. The third measurements were made by Da Costa \& Armandroff (1995), who derived $[\mathrm{Fe} / \mathrm{H}]=-1.99 \pm 0.08$ based on CaII triplet spectroscopy. Our result is compatible with the more metal-rich results.

For alpha-enhancement in this metallicity range, MILES spectra reach to $[\mathrm{Mg} / \mathrm{Fe}]=0.74$, while Coelho is limited to the models of $[\alpha / \mathrm{Fe}]=0.4$. The results based on Coelho $([\alpha / \mathrm{Fe}]=$ $0.24 \pm 0.05$ and $0.21 \pm 0.04)$ are less enhanced than MILES $([\mathrm{Mg} / \mathrm{Fe}]=0.38 \pm 0.06$ and $0.41 \pm 0.04)$, the latter being closer to literature abundance ratios.

\section{Summary and conclusions}

We presented a method of full spectrum fitting, based on the code ETOILE, to derive $v_{\text {helio }}, T_{\text {eff }}, \log (g),[\mathrm{Fe} / \mathrm{H}],[\mathrm{Mg} / \mathrm{Fe}]$, and $[\alpha / \mathrm{Fe}]$ for red giant stars in Milky Way globular clusters. The observations were carried out with FORS2 at VLT/ESO with resolution $R \sim 2000$.

We validated the method using well-known red giant stars covering the parameter space of $4000 \mathrm{~K}<T_{\text {eff }}<6000 \mathrm{~K}, 0.0<$ $\log (g)<4.0,-2.5<[\mathrm{Fe} / \mathrm{H}]<+0.3$, and $-0.2<[\mathrm{Mg} / \mathrm{Fe}]<$ +0.6 . The spectra of these reference stars were taken from the ELODIE library and the parameters from the PASTEL catalogue. The parameters of all stars were recovered by our method. We applied the method to red giant stars, and the code ETOILE has also been applied and validated for dwarf stars by Katz et al. (2011).
To establish the methodology to be adopted for a larger sample of clusters, we chose two metal-rich (NGC 6528, NGC 6553), two moderately metal-rich (M 71, NGC 6558), and two metal-poor clusters (NGC 6426 and Terzan 8). NGC 6528, NGC 6553, and NGC 6558 are located in the bulge, M 71 in the disc, and NGC 6426 and Terzan 8 in the halo. For all clusters the effective temperatures and gravities were clearly determined using the spectral library MILES and the library of Coelho et al. (2005). Metallicities and alpha-element enhancement were also derived, with the caveats that for alpha-enhanced bulge clusters with $[\mathrm{Fe} / \mathrm{H}]>-0.5$, MILES is unsuitable, since it has only solar neighbourhood stars. We therefore used the Coelho results because synthetic libraries have all combinations of parameters. For $[\mathrm{Fe} / \mathrm{H}] \sim-1.0$ MILES only has few stars because there is a lack of such stars in the solar vicinity. For metal-poor clusters, with high $[\alpha / \mathrm{Fe}]$, MILES may be more suitable than Coelho because the latter is limited to $0<[\alpha / \mathrm{Fe}]<0.4 \mathrm{dex}$, if these high $\mathrm{Mg}$ enhancements are confirmed.

Our results agree with the literature parameters available for five of the six template clusters. NGC 6426 was analysed for the first time using spectroscopy of individual stars. Therefore we provide a more precise radial velocity of $-242 \pm 11 \mathrm{~km} \mathrm{~s}^{-1}$, a metallicity $[\mathrm{Fe} / \mathrm{H}]=-2.39 \pm 0.04$, and $[\mathrm{Mg} / \mathrm{Fe}]=0.38 \pm 0.06$. The comparison of our results of $[\mathrm{Fe} / \mathrm{H}]$ and $[\mathrm{Mg} / \mathrm{Fe}]$ with those from field stars from all Galactic components showed that the globular clusters follows the same chemical enrichment pattern as the field stars.

In conclusion, the full spectrum-fitting technique using the code ETOILE together with the MILES and Coelho libraries appears to be suitable for deriving chemical abundances for Milky Way globular clusters from low- and medium-resolution spectra of red giant branch stars. Depending on the stellar population studied, the choice of library with parameter space covering the expected values for the clusters is a crucial ingredient, the observed-spectra library being better for more metal-rich stars and the synthetic-spectra library being preferable for the more metal-poor stars. This method will be applied to the other Milky Way globular clusters from this survey. It is also promising for extragalactic stars, which can be more easily observed with similar resolutions of $R \sim 2000$, and for studies of galaxy formation and evolution.

Acknowledgements. We are grateful to Paula Coelho for useful discussions. B.D. acknowledges financial support from CNPq and ESO. B.B. acknowledges partial financial support from $\mathrm{CNPq}$ and Fapesp.

\section{References}

Allende Prieto, C., Sivarani, T., Beers, T. C., et al. 2008, AJ, 136, 2070 Alves-Brito, A., Barbuy, B., Zoccali, M., et al. 2006, A\&A, 460, 269 Alves-Brito, A., Meléndez, J., Asplund, M., Ramírez, I., \& Yong, D. 2010, A\&A, 513, A35

Appenzeller, I., Fricke, K., Fürtig, W., et al. 1998, The Messenger, 94, 1 Barbuy, B., Zoccali, M., Ortolani, S., et al. 2007, AJ, 134, 1613

Barbuy, B., Zoccali, M., Ortolani, S., et al. 2009, A\&A, 507, 405

Bensby, T., Feltzing, S., Lundström, I., \& Ilyin, I. 2005, A\&A, 433, 185

Bressan, A., Marigo, P., Girardi, L., et al. 2012, MNRAS, 427, 127

Carretta, E., Cohen, J. G., Gratton, R. G., \& Behr, B. B. 2001, AJ, 122, 1469

Carretta, E., Bragaglia, A., Gratton, R., D’Orazi, V., \& Lucatello, S. 2009, A\&A, 508,695

Carretta, E., Bragaglia, A., Gratton, R. G., et al. 2010, A\&A, 516, A55

Carretta, E., Bragaglia, A., Gratton, R. G., et al. 2014, A\&A, 561, A87 Catelan, M., Ferraro, F. R., \& Rood, R. T. 2001, ApJ, 560, 970

Cayrel, R. 1988, in The Impact of Very High S/N Spectroscopy on Stellar Physics, eds. G. Cayrel de Strobel, \& M. Spite, IAU Symp., 132, 345 Cayrel, R., Perrin, M.-N., Barbuy, B., \& Buser, R. 1991, A\&A, 247, 108 Cenarro, A. J., Peletier, R. F., Sánchez-Blázquez, P., et al. 2007, MNRAS, 374, 664 
Coelho, P., Barbuy, B., Meléndez, J., Schiavon, R. P., \& Castilho, B. V. 2005 , A\&A, 443, 735

Cohen, J. G., Gratton, R. G., Behr, B. B., \& Carretta, E. 1999, ApJ, 523, 739

Cohen, J. G., Behr, B. B., \& Briley, M. M. 2001, AJ, 122, 1420

Da Costa, G. S., \& Armandroff, T. E. 1995, AJ, 109, 2533

Da Costa, G. S., Held, E. V., Saviane, I., \& Gullieuszik, M. 2009, ApJ, 705, 1481

de Jong, J. T. A., Yanny, B., Rix, H.-W., et al. 2010, ApJ, 714, 663

Dotter, A., Chaboyer, B., Jevremović, D., et al. 2008, ApJS, 178, 89

Dotter, A., Sarajedini, A., \& Anderson, J. 2011, ApJ, 738, 74

Faber, S. M., Friel, E. D., Burstein, D., \& Gaskell, C. M. 1985, ApJS, 57, 711

Fulbright, J. P. 2000, AJ, 120, 1841

Gilmore, G., Randich, S., Asplund, M., et al. 2012, The Messenger, 147, 25

Gonzalez, O. A., Rejkuba, M., Zoccali, M., et al. 2011, A\&A, 530, A54

Harris, W. E. 1996, AJ, 112, 1487

Hesser, J. E., Shawl, S. J., \& Meyer, J. E. 1986, PASP, 98, 403

Ibata, R. A., Gilmore, G., \& Irwin, M. J. 1994, Nature, 370, 194

Katz, D. 2001, J. Astron. Data, 7, 8

Katz, D., Soubiran, C., Cayrel, R., Adda, M., \& Cautain, R. 1998, A\&A, 338, 151

Katz, D., Soubiran, C., Cayrel, R., et al. 2011, A\&A, 525, A90

Kirby, E. N., Guhathakurta, P., Bolte, M., Sneden, C., \& Geha, M. C. 2009, ApJ, 705, 328

Koleva, M., Prugniel, P., Bouchard, A., \& Wu, Y. 2009, A\&A, 501, 1269

Lee, Y.-W., Demarque, P., \& Zinn, R. 1994, ApJ, 423, 248

Lee, Y. S., Beers, T. C., Sivarani, T., et al. 2008, AJ, 136, 2022

Meléndez, J., \& Cohen, J. G. 2009, ApJ, 699, 2017

Meléndez, J., Barbuy, B., Bica, E., et al. 2003, A\&A, 411, 417

Mészáros, S., Holtzman, J., García Pérez, A. E., et al. 2013, AJ, 146, 133

Milone, A. D. C., Sansom, A. E., \& Sánchez-Blázquez, P. 2011, MNRAS, 414, 1227
Mottini, M., Wallerstein, G., \& McWilliam, A. 2008, AJ, 136, 614 Ortolani, S., Renzini, A., Gilmozzi, R., et al. 1995, Nature, 377, 701 Perryman, M. A. C., de Boer, K. S., Gilmore, G., et al. 2001, A\&A, 369, 339 Pietrinferni, A., Cassisi, S., Salaris, M., \& Castelli, F. 2004, ApJ, 612, 168 Prugniel, P., Soubiran, C., Koleva, M., \& Le Borgne, D. 2007, Vizier Online Data Catalog: III/251 [arXiv: astro-ph/0703658]

Ramírez, S. V., \& Cohen, J. G. 2002, AJ, 123, 3277

Ramírez, S. V., Cohen, J. G., Buss, J., \& Briley, M. M. 2001, AJ, 122, 1429

Reddy, B. E., Lambert, D. L., \& Allende Prieto, C. 2006, MNRAS, 367, 1329

Roederer, I. U. 2009, AJ, 137, 272

Saito, R. K., Zoccali, M., McWilliam, A., et al. 2011, AJ, 142, 76

Sánchez Almeida, J., \& Allende Prieto, C. 2013, ApJ, 763, 50

Sánchez-Blázquez, P., Peletier, R. F., Jiménez-Vicente, J., et al. 2006, MNRAS, 371,703

Saviane, I., da Costa, G. S., Held, E. V., et al. 2012, A\&A, 540, A27

Soubiran, C., Le Campion, J.-F., Cayrel de Strobel, G., \& Caillo, A. 2010, A\&A, 515, A111

Steinmetz, M., Zwitter, T., Siebert, A., et al. 2006, AJ, 132, 1645

VandenBerg, D. A., Brogaard, K., Leaman, R., \& Casagrande, L. 2013, ApJ, 775,134

Worthey, G., Faber, S. M., Gonzalez, J. J., \& Burstein, D. 1994, ApJS, 94, 687

Wu, Y., Luo, A.-L., Li, H.-N., et al. 2011, Res. Astron. Astrophys., 11, 924

Wylie-de Boer, E., \& Freeman, K. 2010, in IAU Symp. 262, eds. G. R. Bruzual, \& S. Charlot, 448

York, D. G., Adelman, J., Anderson, Jr., J. E., et al. 2000, AJ, 120, 1579

Zinn, R., \& West, M. J. 1984, ApJS, 55, 45

Zoccali, M., Renzini, A., Ortolani, S., Bica, E., \& Barbuy, B. 2001, AJ, 121, 2638

Zoccali, M., Barbuy, B., Hill, V., et al. 2004, A\&A, 423, 507

Zoccali, M., Hill, V., Lecureur, A., et al. 2008, A\&A, 486, 177 
B. Dias et al.: FORS2/VLT survey of Milky Way globular clusters. I.

M 71

NGC 6553
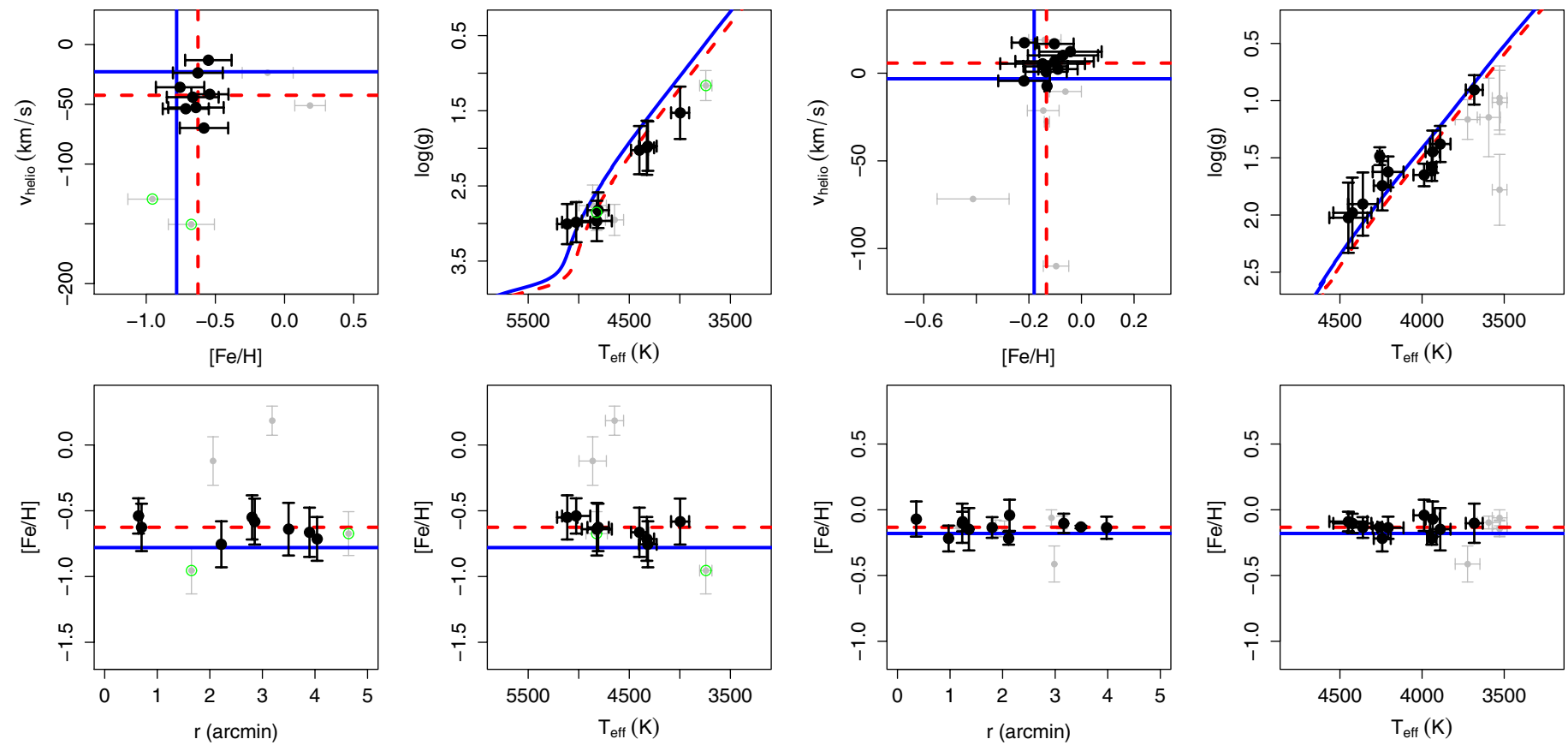

Fig. 14. Same as Fig. 13 for M 71 .

Fig. 16. Same as Fig. 13 for NGC 6553.

NGC 6426
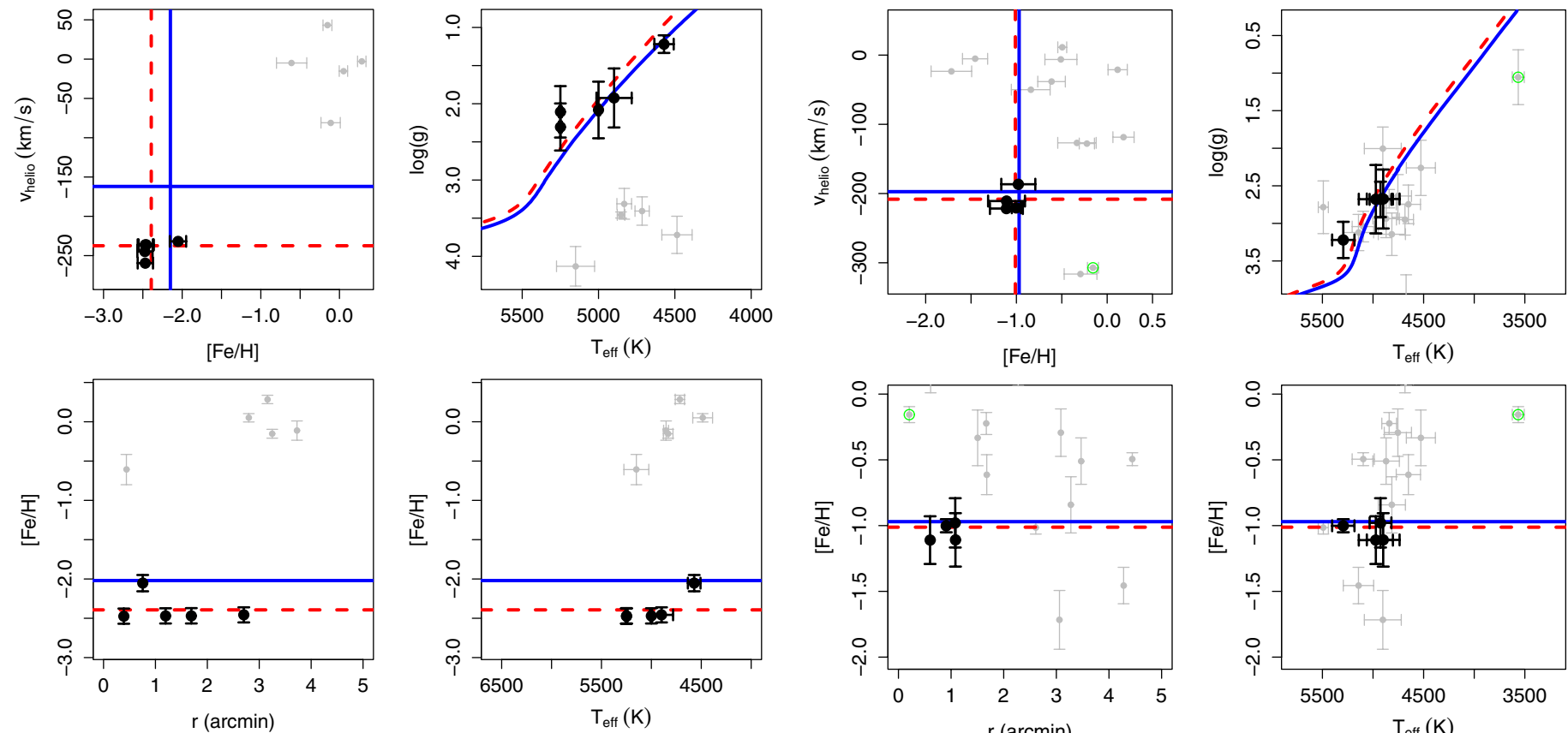

Fig. 15. Same as Fig. 13 for NGC 6426.
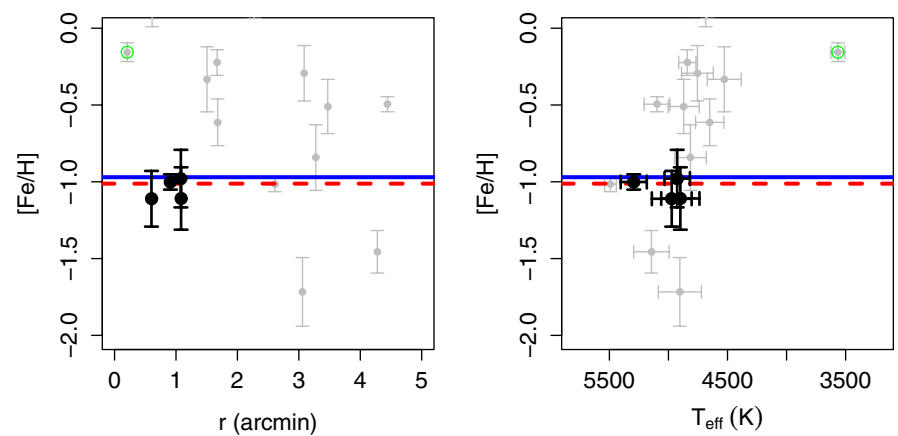

Fig. 17. Same as Fig. 13 for NGC 6558. 
Terzan 8
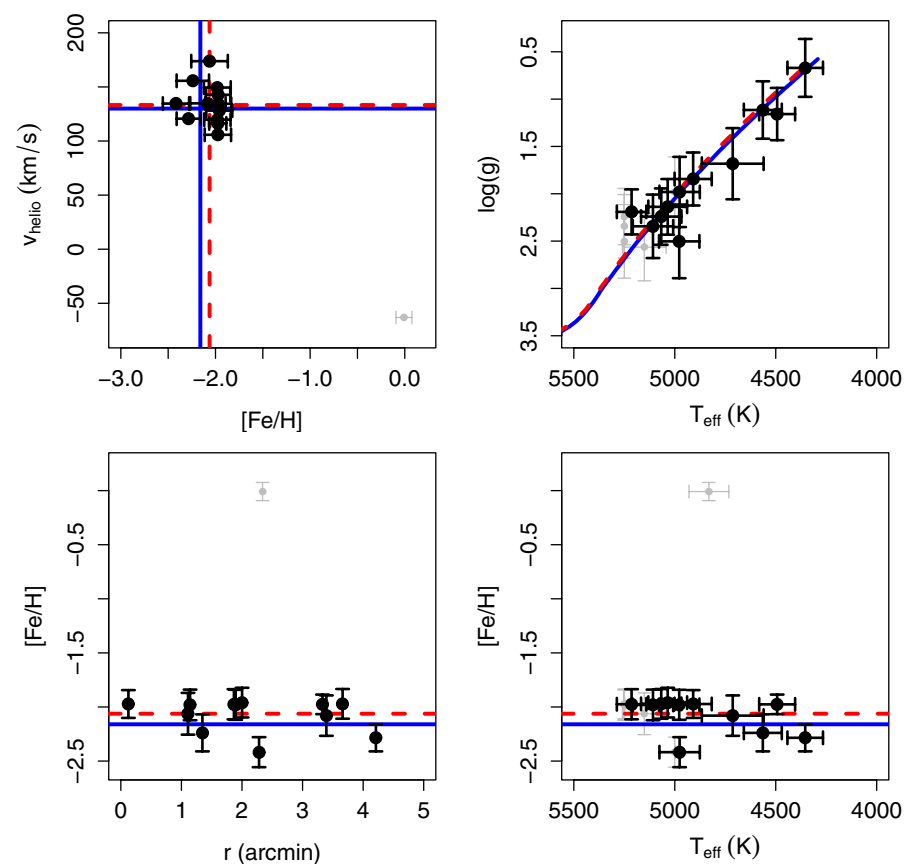

Fig. 18. Same as Fig. 13 for Terzan 8 . 
B. Dias et al.: FORS2/VLT survey of Milky Way globular clusters. I.

Table 3. Star-by-star coordinates, magnitude, colour, heliocentric radial velocity, and membership selection.

\begin{tabular}{|c|c|c|c|c|c|c|c|}
\hline Star ID & $\begin{array}{l}\text { RA (J2000) } \\
(\operatorname{deg})\end{array}$ & $\begin{array}{l}\operatorname{Dec}(\mathrm{J} 2000) \\
(\operatorname{deg})\end{array}$ & $\begin{array}{c}V \\
(\mathrm{mag})\end{array}$ & $\begin{array}{l}V-I \\
(\mathrm{mag})\end{array}$ & $\begin{array}{c}v_{\text {helio }} \\
\left(\mathrm{km} \mathrm{s}^{-1}\right)\end{array}$ & $\begin{array}{l}v_{\text {helio-CaT }} \\
\left(\mathrm{km} \mathrm{s}^{-1}\right)\end{array}$ & Members \\
\hline NGC 6528_2 & 271.1807715771910 & -30.0070234836130 & 15.987 & 1.982 & -51.55 & - & \\
\hline NGC 6528_3 & 271.1933667526377 & -30.0114575457940 & 15.647 & 2.836 & 61.28 & - & \\
\hline NGC 6528_4 & 271.1789935185810 & -30.0172537974630 & 15.596 & 2.257 & -314.24 & - & \\
\hline NGC 6528_5 & 271.1883528713980 & -30.0237445060760 & 15.939 & 1.937 & -226.44 & - & \\
\hline NGC 6528_6 & 271.1974416677920 & -30.0295599638440 & 17.060 & 1.741 & -4.93 & - & \\
\hline NGC 6528_7 & 271.2102328036860 & -30.0372195159180 & 15.887 & 2.140 & -79.24 & - & \\
\hline NGC 6528_8 & 271.2027175539120 & -30.0434602130820 & 16.428 & 1.831 & 179.09 & 200 & M \\
\hline NGC 6528_9 & 271.1969387171677 & -30.0500739849720 & 16.501 & 1.774 & 199.18 & 208 & $\mathrm{M}$ \\
\hline NGC 6528_10 & 271.2070341687930 & -30.0537176170110 & 16.145 & 2.007 & 177.87 & 209 & M \\
\hline NGC 6528_11 & 271.2013524700317 & -30.0600904185180 & 15.511 & 2.255 & 182.67 & 202 & M \\
\hline NGC 6528_13 & 271.1879324598030 & -30.0747465095010 & 16.429 & 1.731 & -29.84 & - & \\
\hline NGC 6528_14 & 271.2012738946260 & -30.0802412070160 & 16.032 & 2.056 & -40.56 & - & \\
\hline NGC 6528_15 & 271.1774205888063 & -30.0879237445260 & 15.892 & 2.062 & -288.86 & - & \\
\hline NGC 6528_16 & 271.1843122189650 & -30.0926974725130 & 15.954 & 2.150 & -104.91 & - & \\
\hline NGC 6528_17 & 271.1904270294383 & -30.1021087576640 & 16.537 & 1.844 & -62.96 & - & \\
\hline NGC 6528_18 & 271.1726747862950 & -30.1092237523470 & 16.666 & 1.767 & -212.87 & - & \\
\hline NGC 6528_19 & 271.1832358189100 & -30.1108940656500 & 16.468 & 1.778 & -54.47 & - & \\
\hline NGC 6553_1 & 272.3536106669400 & -25.8497112721910 & 15.816 & 2.109 & 3.90 & -8 & M \\
\hline NGC 6553_3 & 272.3507085422689 & -25.8636412811220 & 15.832 & 2.010 & 16.71 & 19 & M \\
\hline NGC 6553_4 & 272.2982167426244 & -25.8658039783470 & 15.310 & 2.618 & -71.71 & -39 & M \\
\hline NGC 6553_5 & 272.3267021788947 & -25.8733214097360 & 15.775 & 2.522 & 12.23 & -12 & $\mathrm{M}$ \\
\hline NGC 6553_6 & 272.3161974470756 & -25.8795540059450 & 16.237 & 2.177 & 0.81 & -6 & M \\
\hline NGC 6553_7 & 272.3258216397713 & -25.8883122417460 & 15.338 & 2.881 & 6.76 & 3 & M \\
\hline NGC 6553_8 & 272.3501542761960 & -25.8928074210270 & 15.370 & 3.088 & 18.98 & - & \\
\hline NGC 6553_9 & 272.3409674217040 & -25.9007568498840 & 16.253 & 2.097 & -27.72 & -32 & M \\
\hline NGC 6553_10 & 272.3439213760840 & -25.9071903257530 & 15.985 & 1.998 & 2.34 & 2 & M \\
\hline NGC 6553_11 & 272.3287891424960 & -25.9110912319810 & 15.441 & 2.443 & 10.12 & -3 & M \\
\hline NGC 6553_13 & 272.3235733284760 & -25.9249134402580 & 15.906 & 2.075 & -4.38 & -12 & M \\
\hline NGC 6553_14 & 272.3157993633750 & -25.9300477560180 & 15.187 & 2.382 & 5.40 & -5 & M \\
\hline NGC 6553_15 & 272.3047106358403 & -25.9370597367310 & 14.980 & 2.996 & -21.36 & - & \\
\hline NGC 6553_16 & 272.3280756666470 & -25.9436286781210 & 15.708 & 2.332 & 17.42 & 8 & M \\
\hline NGC 6553_17 & 272.2895515368990 & -25.9484055025060 & 15.065 & 2.849 & -110.00 & - & \\
\hline NGC 6553_18 & 272.3144982937293 & -25.9566926017970 & 15.407 & 3.681 & -10.44 & - & \\
\hline NGC 6553_19 & 272.3440933085433 & -25.9630097530170 & 15.843 & 2.209 & -7.38 & -4 & M \\
\hline M71_2 & 298.4578984488130 & 18.8301452486084 & 14.606 & 1.269 & -150.46 & -34 & M \\
\hline M71_4 & 298.4609554670447 & 18.81 & 13.030 & 1.503 & -53.75 & -41 & M \\
\hline M71_5 & 298.4632902665389 & 18.8090499016740 & 14.391 & 1.282 & -52.74 & -26 & M \\
\hline M71_6 & 298.4900822754500 & 18.7995866372160 & 13.534 & 1.367 & -44.07 & -37 & M \\
\hline M71_7 & 298.4510869475747 & 18.8011235588184 & 12.376 & 1.719 & -69.84 & -34 & M \\
\hline M71_8 & 298.4854008510480 & 18.7869940682184 & 14.421 & 1.280 & -51.13 & - & \\
\hline M71_9 & 298.4422411559920 & 18.7910962573810 & 13.146 & 1.530 & -35.78 & -24 & M \\
\hline M71_10 & 298.4476878071520 & 18.7813713381542 & 12.140 & 2.042 & -129.33 & -26 & M \\
\hline M71_13 & 298.4496765965810 & 18.7641970806387 & 14.281 & 1.303 & -23.72 & -5 & M \\
\hline M71_14 & 298.4902210311380 & 18.7496150618418 & 14.582 & 1.210 & -13.11 & -17 & M \\
\hline M71_15 & 298.4521026881480 & 18.7474809643955 & 14.580 & 1.209 & -41.62 & -8 & M \\
\hline M71_16 & 298.4732927595957 & 18.7366583486676 & 14.727 & 1.231 & -23.67 & - & \\
\hline NGC 6558_3 & 2.6225545017980 & -31.7335169754310 & 6.862 & 1.434 & -6.32 & - & \\
\hline NGC 6558_4 & 272.5685475821600 & -31.7363376028500 & 16.541 & 1.441 & -38.29 & - & \\
\hline NGC 6558_5 & 272.6214120279947 & -31.7468917846890 & 16.349 & 1.360 & -23.46 & - & \\
\hline NGC 6558_6 & 272.5796695743913 & -31.7469946267620 & 15.982 & 1.524 & -210.85 & -196 & M \\
\hline NGC 6558_7 & 272.5899712149563 & -31.7570504471050 & 15.803 & 1.393 & -186.59 & -187 & M \\
\hline NGC 6558_8 & 272.5739816190383 & -31.7605125893880 & 13.651 & 2.044 & -307.26 & -210 & M \\
\hline NGC 6558_9 & 272.5637020636180 & -31.7666306345590 & 16.026 & 1.499 & -221.66 & -204 & M \\
\hline NGC 6558_10 & 272.5771880085779 & -31.7733227718450 & 16.710 & 1.580 & -21.13 & - & \\
\hline NGC 6558_11 & 272.5757815235553 & -31.7788392433250 & 16.753 & 1.329 & -220.56 & -195 & M \\
\hline NGC 6558_12 & 272.5805489015007 & -31.7879129654280 & 16.521 & 1.452 & -126.79 & - & \\
\hline NGC 6558_13 & 272.5717825299750 & -31.7916739864870 & 15.626 & 1.477 & -127.74 & - & \\
\hline NGC 6558_14 & 272.5809044425367 & -31.8010529051890 & 16.740 & 1.188 & -118.59 & - & \\
\hline NGC 6558_15 & 272.5652668675210 & -31.8066271885810 & 16.480 & 1.293 & 105.58 & - & \\
\hline NGC 6558_16 & 272.5564861827613 & -31.8124812867920 & 16.366 & 1.450 & -316.32 & - & \\
\hline
\end{tabular}

Notes. The velocities from CaT were taken from from Saviane et al. (2012) for NGC 6528, NGC 6553, M 71, and NGC 6558, and from Vasquez et al. (in prep.) for NGC 6426 and Terzan 8. 
Table 3. continued.

\begin{tabular}{|c|c|c|c|c|c|c|c|}
\hline Star ID & $\begin{array}{l}\text { RA (J2000) } \\
(\mathrm{deg})\end{array}$ & $\begin{array}{l}\operatorname{Dec}(\mathrm{J} 2000) \\
(\mathrm{deg})\end{array}$ & $\begin{array}{c}V \\
(\mathrm{mag})\end{array}$ & $\begin{array}{l}V-I \\
(\mathrm{mag})\end{array}$ & $\begin{array}{c}v_{\text {helio }} \\
\left(\mathrm{km} \mathrm{s}^{-1}\right)\end{array}$ & $\begin{array}{l}v_{\text {helio-CaT }} \\
\left(\mathrm{km} \mathrm{s}^{-1}\right)\end{array}$ & Members \\
\hline NGC 6558_17 & 272.5801632324050 & -31.8180552937480 & 16.964 & 1.348 & -49.93 & - & \\
\hline NGC 6558 & 272.6131914789087 & -31.8262981571080 & 16.911 & 1.288 & 11.27 & - & \\
\hline NGC 6558_19 & 272.5939188278727 & -31.8321512784620 & 17.013 & 1.299 & -5.38 & - & \\
\hline NGC 6426_1 & 266.2553357204670 & 3.2257821720617 & 18.018 & 1.359 & -80.97 & -49.927 & \\
\hline NGC 64262 & 266.2088188480099 & 3.2192772570129 & 17.597 & 1.394 & -2.85 & 11.325 & \\
\hline NGC 6426_3 & 266.2200960712349 & 3.2161573577027 & 16.468 & 1.586 & -15.18 & 12.662 & \\
\hline NGC 6426_4 & 266.2059346355020 & 3.2095717676480 & 16.072 & 1.644 & -236.29 & -230.019 & M \\
\hline NGC 6426_7 & 266.2472044411759 & 3.1904905620518 & 17.666 & 1.459 & -259.55 & -225.413 & M \\
\hline NGC 6426_9 & 266.2324246578110 & 3.1746245634344 & 17.170 & 1.515 & -244.78 & -222.660 & $\mathrm{M}$ \\
\hline NGC 6426_10 & 266.2152297889280 & 3.1685396261324 & 15.544 & 1.749 & -231.87 & -221.297 & M \\
\hline NGC 6426_11 & 266.2225277929239 & 3.1649406775723 & 17.892 & 1.354 & -4.92 & 12.270 & \\
\hline NGC 6426 13 & 266.2203523684530 & 3.1516328071727 & 16.745 & 1.482 & -236.15 & -225.730 & $\mathrm{M}$ \\
\hline NGC 6426_18 & 266.2405437043161 & 3.1175053813749 & 16.970 & 1.434 & 43.08 & 72.570 & \\
\hline & & & 16.706 & 1.232 & 134.4 & & $\mathrm{M}$ \\
\hline Terzan8_4 & 9075670249 & 4796670 & 15.268 & 1.436 & 120 & 137.4998 & M \\
\hline Terzan8_5 & 295.4297741922069 & -33.9618073863440 & 17.002 & 1.135 & 134 & 152.6001 & M \\
\hline Terzan8_6 & 295.4324851623650 & -33.9679803480730 & 17.517 & 1.076 & 119.81 & 138.3819 & M \\
\hline Terzan8_8 & 295.4284871648290 & -33.9821965410250 & 17.089 & 1.108 & 173.81 & 150.6620 & M \\
\hline Terzan8_9 & 295.4571652028810 & -33.9956864225960 & 15.447 & 1.352 & 155.67 & 139.3673 & M \\
\hline Terzan8_10 & 295.4738836006140 & -34.0028288269560 & 17.381 & 1.076 & -63.09 & -25.6758 & \\
\hline Terzan8_11 & 295.4369091804230 & -34.0003943267700 & 15.682 & 1.277 & 142.74 & 150.6086 & M \\
\hline Terzan8_13 & 295.4233824419659 & -34.0145360437400 & 17.364 & 1.101 & 149.42 & 144.2031 & M \\
\hline Terzan8_14 & 295.4815071780459 & -34.0298070702590 & 15.530 & 1.388 & 116.26 & 131.9558 & M \\
\hline Terzan8 15 & 295.4375324314209 & -34.0304794278020 & 16.433 & 1.219 & 105.77 & 121.5507 & $\mathrm{M}$ \\
\hline Terzan8 8 & 295.4285209120099 & -34.0321898355090 & 16.778 & 1.122 & 128.12 & 140.9906 & $\mathrm{M}$ \\
\hline Terzan8_18 & 295.4529047094940 & -34.0531331777060 & 16.143 & 1.273 & 134.81 & 131.1500 & M \\
\hline
\end{tabular}


B. Dias et al.: FORS2/VLT survey of Milky Way globular clusters. I.

Table 4. List of 49 well-known stars selected from ELODIE spectral library.

\begin{tabular}{|c|c|c|c|c|c|c|c|c|c|c|c|}
\hline Elodie & Star & $R R_{\text {tot }}$ & $N$ & $S_{\lim }$ & $\begin{array}{l}T_{\text {eff }}(\mathrm{K}) \\
\text { (literature) }\end{array}$ & $\begin{array}{c}\log (g) \\
\text { (literature) }\end{array}$ & $\begin{array}{r}{[\mathrm{Fe} / \mathrm{H}]} \\
\text { (literature) }\end{array}$ & $\begin{array}{l}T_{\text {eff }}(\mathrm{K}) \\
\text { (this work) }\end{array}$ & $\begin{array}{c}\log g \\
\text { (this work) }\end{array}$ & $\begin{array}{r}{[\mathrm{Fe} / \mathrm{H}]} \\
\text { (this work) }\end{array}$ & $\begin{array}{r}{[\mathrm{Mg} / \mathrm{Fe}]} \\
\text { (this work) }\end{array}$ \\
\hline 1 & HD 000245 & 0.107 & 2 & 1.042 & $5490 \pm 153$ & $3.48 \pm 0.15$ & $-0.77 \pm 0.08$ & $5378 \pm 60$ & $3.67 \pm 0.06$ & $-0.84 \pm 0.20$ & $0.34 \pm 0.08$ \\
\hline 9 & HD 002796 & 0.062 & 1 & 1.000 & $4931 \pm 60$ & $1.45 \pm 0.34$ & $-2.32 \pm 0.11$ & $4945 \pm 133$ & $1.36 \pm 0.08$ & $-2.31 \pm 0.11$ & $0.37 \pm 0.08$ \\
\hline 19 & HD 004395 & 0.042 & 5 & 1.116 & $5487 \pm 38$ & $3.33 \pm 0.05$ & $-0.35 \pm 0.04$ & $5330 \pm 149$ & $3.24 \pm 0.16$ & $-0.34 \pm 0.11$ & $0.18 \pm 0.07$ \\
\hline 31 & HD 006833 & 0.092 & 1 & 1.000 & $4426 \pm 95$ & $1.28 \pm 0.32$ & $-0.91 \pm 0.14$ & $4380 \pm 217$ & $1.25 \pm 0.64$ & $-0.99 \pm 0.29$ & $0.30 \pm 0.11$ \\
\hline 32 & HD 006920 & 0.035 & 8 & 1.319 & $5886 \pm 111$ & $3.60 \pm 0.28$ & $-0.10 \pm 0.09$ & $5854 \pm 100$ & $3.60 \pm 0.05$ & $-0.10 \pm 0.14$ & $0.10 \pm 0.09$ \\
\hline 33 & HD 008724 & 0.018 & 2 & 1.001 & $4586 \pm 84$ & $1.39 \pm 0.26$ & $-1.73 \pm 0.13$ & $4626 \pm 6$ & $1.40 \pm 0.06$ & $-1.75 \pm 0.00$ & $0.37 \pm 0.08$ \\
\hline 47 & HD 013530 & 0.013 & 6 & 1.036 & $4772 \pm 106$ & $2.60 \pm 0.39$ & $-0.54 \pm 0.11$ & $4769 \pm 87$ & $2.63 \pm 0.21$ & $-0.54 \pm 0.15$ & $0.37 \pm 0.08$ \\
\hline 66 & HD 015596 & 0.047 & 9 & 1.211 & $4808 \pm 59$ & $2.66 \pm 0.32$ & $-0.65 \pm 0.06$ & $4760 \pm 58$ & $2.54 \pm 0.14$ & $-0.66 \pm 0.07$ & $0.40 \pm 0.06$ \\
\hline 67 & HD 015596 & 0.037 & 10 & 1.298 & $4808 \pm 59$ & $2.66 \pm 0.32$ & $-0.65 \pm 0.06$ & $4751 \pm 52$ & $2.57 \pm 0.12$ & $-0.64 \pm 0.06$ & $0.37 \pm 0.07$ \\
\hline 68 & HD 015596 & 0.047 & 9 & 1.248 & $4808 \pm 59$ & $2.66 \pm 0.32$ & $-0.65 \pm 0.06$ & $4759 \pm 56$ & $2.54 \pm 0.13$ & $-0.66 \pm 0.06$ & $0.40 \pm 0.06$ \\
\hline 69 & HD 016458 & 0.118 & 2 & 1.115 & $4593 \pm 123$ & $1.84 \pm 0.26$ & $-0.35 \pm 0.05$ & $4992 \pm 513$ & $1.97 \pm 0.95$ & $-0.34 \pm 0.25$ & $0.33 \pm 0.23$ \\
\hline 88 & HD 020512 & 0.086 & 3 & 1.118 & $5212 \pm 63$ & $3.65 \pm 0.14$ & $-0.22 \pm 0.19$ & $5074 \pm 38$ & $3.35 \pm 0.14$ & $-0.22 \pm 0.20$ & $0.12 \pm 0.03$ \\
\hline 89 & HD 020512 & 0.094 & 3 & 1.101 & $5212 \pm 63$ & $3.65 \pm 0.14$ & $-0.22 \pm 0.19$ & $5074 \pm 39$ & $3.35 \pm 0.15$ & $-0.21 \pm 0.21$ & $0.12 \pm 0.03$ \\
\hline 117 & HD 026297 & 0.133 & 11 & 1.263 & $4445 \pm 140$ & $1.02 \pm 0.28$ & $-1.74 \pm 0.15$ & $4460 \pm 73$ & $1.15 \pm 0.19$ & $-1.66 \pm 0.08$ & $0.48 \pm 0.04$ \\
\hline 151 & HD 035369 & 0.032 & 6 & 1.062 & $4885 \pm 112$ & $2.57 \pm 0.27$ & $-0.21 \pm 0.08$ & $4900 \pm 45$ & $2.65 \pm 0.08$ & $-0.21 \pm 0.05$ & $0.06 \pm 0.02$ \\
\hline 227 & HD 045282 & 0.023 & 1 & 1.000 & $5264 \pm 86$ & $3.19 \pm 0.16$ & $-1.43 \pm 0.12$ & $5348 \pm 110$ & $3.24 \pm 0.29$ & $-1.44 \pm 0.05$ & $0.22 \pm 0.07$ \\
\hline 228 & HD 045282 & 0.065 & 3 & 1.042 & $5264 \pm 86$ & $3.19 \pm 0.16$ & $-1.43 \pm 0.12$ & $5268 \pm 48$ & $3.14 \pm 0.23$ & $-1.52 \pm 0.05$ & $0.36 \pm 0.06$ \\
\hline 253 & HD 046480 & 0.016 & 15 & 1.218 & $4785 \pm 26$ & $2.63 \pm 0.12$ & $-0.49 \pm 0.01$ & $4791 \pm 55$ & $2.65 \pm 0.13$ & $-0.50 \pm 0.08$ & $0.31 \pm 0.05$ \\
\hline 254 & HD 046480 & 0.016 & 15 & 1.214 & $4785 \pm 26$ & $2.63 \pm 0.12$ & $-0.49 \pm 0.01$ & $4791 \pm 55$ & $2.65 \pm 0.13$ & $-0.50 \pm 0.08$ & $0.31 \pm 0.05$ \\
\hline 314 & HD 063791 & 0.040 & 4 & 1.028 & $4715 \pm 78$ & $1.75 \pm 0.08$ & $-1.68 \pm 0.08$ & $4868 \pm 275$ & $1.78 \pm 0.47$ & $-1.66 \pm 0.38$ & $0.44 \pm 0.12$ \\
\hline 384 & HD 087140 & 0.033 & 2 & 1.038 & $5129 \pm 103$ & $2.66 \pm 0.25$ & $-1.80 \pm 0.13$ & $5090 \pm 5$ & $2.58 \pm 0.10$ & $-1.82 \pm 0.06$ & $0.42 \pm 0.01$ \\
\hline 425 & HD 108317 & 0.039 & 2 & 1.034 & $5259 \pm 111$ & $2.68 \pm 0.25$ & $-2.27 \pm 0.05$ & $5117 \pm 40$ & $2.70 \pm 0.15$ & $-2.33 \pm 0.11$ & $0.45 \pm 0.08$ \\
\hline 452 & HD 117876 & 0.075 & 3 & 1.091 & $4747 \pm 128$ & $2.27 \pm 0.03$ & $-0.48 \pm 0.02$ & $4806 \pm 18$ & $2.25 \pm 0.15$ & $-0.44 \pm 0.03$ & $0.42 \pm 0.10$ \\
\hline 454 & HD 122956 & 0.023 & 3 & 1.018 & $4633 \pm 78$ & $1.46 \pm 0.18$ & $-1.72 \pm 0.11$ & $4646 \pm 16$ & $1.43 \pm 0.04$ & $-1.73 \pm 0.02$ & $0.42 \pm 0.07$ \\
\hline 459 & HD 124897 & 0.136 & 5 & 1.023 & $4302 \pm 115$ & $1.66 \pm 0.31$ & $-0.52 \pm 0.11$ & $4346 \pm 98$ & $1.87 \pm 0.46$ & $-0.55 \pm 0.24$ & $0.29 \pm 0.15$ \\
\hline 470 & HD 135722 & 0.011 & 3 & 1.016 & & $2.60 \pm 0.41$ & $-0.40 \pm 0.10$ & & $2.60 \pm 0.03$ & $-0.40 \pm 0.03$ & $0.14 \pm 0.04$ \\
\hline 473 & HD 137759 & 0.142 & 2 & 1.014 & $4549 \pm 118$ & $2.88 \pm 0.21$ & $0.13 \pm 0.11$ & $4558 \pm 64$ & $2.54 \pm 0.07$ & $0.14 \pm 0.02$ & $-0.03 \pm 0.11$ \\
\hline 509 & HD 159181 & 0.180 & 4 & 1.215 & $5234 \pm 158$ & $1.56 \pm 0.19$ & $0.15 \pm 0.12$ & $5235 \pm 48$ & $1.82 \pm 0.31$ & $0.14 \pm 0.24$ & $0.04 \pm 0.19$ \\
\hline 566 & HD 166161 & 0.059 & 2 & 1.040 & $5210 \pm 167$ & $2.25 \pm 0.42$ & $-1.22 \pm 0.13$ & $5071 \pm 122$ & $2.16 \pm 0.11$ & $-1.18 \pm 0.05$ & $0.31 \pm 0.06$ \\
\hline 568 & HD 166208 & 0.161 & 1 & 1.000 & & $2.71 \pm 0.08$ & $0.07 \pm 0.11$ & & $2.52 \pm 0.05$ & $0.08 \pm 0.04$ & $0.17 \pm 0.01$ \\
\hline 652 & HD 175305 & 0.082 & 1 & 1.000 & $5053 \pm 140$ & $2.49 \pm 0.26$ & $-1.43 \pm 0.07$ & $4899 \pm 17$ & $2.30 \pm 0.03$ & $-1.43 \pm 0.05$ & $0.27 \pm 0.06$ \\
\hline 701 & HD 187111 & 0.125 & 4 & 1.149 & $4299 \pm 75$ & $0.74 \pm 0.30$ & $-1.78 \pm 0.18$ & $4343 \pm 77$ & $0.79 \pm 0.21$ & $-1.59 \pm 0.09$ & $0.47 \pm 0.04$ \\
\hline 763 & HD 198149 & 0.078 & 2 & 1.015 & $4956 \pm 177$ & $3.35 \pm 0.22$ & $-0.12 \pm 0.18$ & $5027 \pm 10$ & $3.12 \pm 0.06$ & $-0.12 \pm 0.06$ & $0.12 \pm 0.01$ \\
\hline 791 & HD 204543 & 0.026 & 1 & 1.000 & $4667 \pm 68$ & $1.30 \pm 0.23$ & $-1.80 \pm 0.10$ & $4617 \pm 43$ & $1.31 \pm 0.08$ & $-1.76 \pm 0.10$ & $0.24 \pm 0.07$ \\
\hline 792 & HD 204613 & 0.101 & 2 & 1.208 & $5742 \pm 135$ & $3.72 \pm 0.19$ & $-0.51 \pm 0.16$ & $5614 \pm 111$ & $3.45 \pm 0.09$ & $-0.48 \pm 0.08$ & $0.21 \pm 0.15$ \\
\hline 803 & HD 207130 & 0.093 & 3 & 1.011 & $4760 \pm 53$ & $2.63 \pm 0.15$ & $0.01 \pm 0.11$ & $4727 \pm 16$ & $2.40 \pm 0.12$ & $0.01 \pm 0.03$ & $0.07 \pm 0.01$ \\
\hline 825 & HD 216143 & 0.045 & 2 & 1.019 & $4495 \pm 82$ & $1.12 \pm 0.38$ & $-2.20 \pm 0.06$ & $4480 \pm 8$ & $1.15 \pm 0.06$ & $-2.12 \pm 0.01$ & $0.39 \pm 0.05$ \\
\hline 826 & HD 216174 & 0.066 & 2 & 1.145 & $4413 \pm 23$ & $2.11 \pm 0.36$ & $-0.55 \pm 0.02$ & $4381 \pm 9$ & $2.21 \pm 0.02$ & $-0.53 \pm 0.00$ & $0.33 \pm 0.04$ \\
\hline 836 & HD 218857 & 0.054 & 8 & 1.098 & $5119 \pm 44$ & $2.50 \pm 0.37$ & $-1.91 \pm 0.09$ & $5067 \pm 89$ & $2.37 \pm 0.31$ & $-1.93 \pm 0.15$ & $0.41 \pm 0.04$ \\
\hline 848 & HD 221345 & 0.019 & 12 & 1.224 & $4635 \pm 108$ & $2.49 \pm 0.32$ & $-0.30 \pm 0.07$ & $4666 \pm 45$ & $2.50 \pm 0.09$ & $-0.30 \pm 0.04$ & $0.18 \pm 0.07$ \\
\hline 849 & HD 221377 & 0.182 & 3 & 1.150 & $6176 \pm 188$ & $3.61 \pm 0.17$ & $-0.88 \pm 0.17$ & $6027 \pm 57$ & $3.24 \pm 0.15$ & $-1.01 \pm 0.15$ & $0.57 \pm 0.12$ \\
\hline 871 & HD 232078 & 0.101 & 1 & 1.000 & $3939 \pm 175$ & $0.31 \pm 0.34$ & $-1.58 \pm 0.15$ & $3983 \pm 186$ & $0.30 \pm 0.53$ & $-1.73 \pm 0.76$ & $0.27 \pm 0.15$ \\
\hline 878 & $\mathrm{BD}+233130$ & 0.033 & 1 & 1.000 & $5119 \pm 140$ & $2.39 \pm 0.38$ & $-2.62 \pm 0.19$ & $5039 \pm 20$ & $2.42 \pm 0.16$ & $-2.55 \pm 0.01$ & $0.60 \pm 0.04$ \\
\hline 883 & $\mathrm{BD}+302611$ & 0.139 & 2 & 1.013 & $4292 \pm 100$ & $0.96 \pm 0.37$ & $-1.41 \pm 0.19$ & $4421 \pm 274$ & $0.83 \pm 0.72$ & $-1.43 \pm 0.05$ & $0.46 \pm 0.13$ \\
\hline 927 & HD 000245 & 0.110 & 2 & 1.032 & $5490 \pm 153$ & $3.48 \pm 0.15$ & $-0.77 \pm 0.08$ & $5377 \pm 59$ & $3.67 \pm 0.06$ & $-0.84 \pm 0.19$ & $0.34 \pm 0.08$ \\
\hline 941 & HD 003546 & 0.035 & 15 & 1.412 & $4906 \pm 168$ & $2.45 \pm 0.43$ & $-0.64 \pm 0.12$ & $4868 \pm 71$ & $2.51 \pm 0.19$ & $-0.65 \pm 0.09$ & $0.27 \pm 0.03$ \\
\hline 1395 & HD 105546 & 0.047 & 2 & 1.018 & $5234 \pm 79$ & $2.38 \pm 0.14$ & $-1.39 \pm 0.15$ & $5387 \pm 190$ & $2.30 \pm 0.14$ & $-1.37 \pm 0.23$ & $0.54 \pm 0.06$ \\
\hline 1452 & HD 122563 & 0.043 & 1 & 1.000 & $4565 \pm 131$ & $1.17 \pm 0.24$ & $-2.62 \pm 0.14$ & $4566 \pm 440$ & $1.12 \pm 1.31$ & $-2.63 \pm 0.37$ & $0.60 \pm 0.22$ \\
\hline 1483 & HD 136512 & 0.098 & 2 & 1.060 & $4719 \pm 66$ & $2.72 \pm 0.04$ & $-0.33 \pm 0.16$ & $4747 \pm 46$ & $2.62 \pm 0.25$ & $-0.30 \pm 0.00$ & $0.08 \pm 0.03$ \\
\hline 1484 & HD 136512 & 0.110 & 4 & 1.072 & $4719 \pm 66$ & $2.72 \pm 0.04$ & $-0.33 \pm 0.16$ & $4754 \pm 29$ & $2.53 \pm 0.17$ & $-0.30 \pm 0.08$ & $0.14 \pm 0.11$ \\
\hline 1485 & HD 136512 & 0.111 & 4 & 1.072 & $4719 \pm 66$ & $2.72 \pm 0.04$ & $-0.33 \pm 0.16$ & $4754 \pm 29$ & $2.53 \pm 0.17$ & $-0.30 \pm 0.08$ & $0.14 \pm 0.11$ \\
\hline 1486 & HD 136512 & 0.070 & 14 & 1.149 & $4719 \pm 66$ & $2.72 \pm 0.04$ & $-0.33 \pm 0.16$ & $4824 \pm 25$ & $2.54 \pm 0.08$ & $-0.33 \pm 0.05$ & $0.21 \pm 0.06$ \\
\hline 1487 & HD 136512 & 0.080 & 13 & 1.144 & $4719 \pm 66$ & $2.72 \pm 0.04$ & $-0.33 \pm 0.16$ & $4821 \pm 27$ & $2.52 \pm 0.08$ & $-0.32 \pm 0.06$ & $0.21 \pm 0.06$ \\
\hline 1576 & HD 162211 & 0.058 & 4 & 1.210 & $4568 \pm 74$ & $2.74 \pm 0.11$ & $0.04 \pm 0.06$ & $4581 \pm 72$ & $2.59 \pm 0.17$ & $0.04 \pm 0.02$ & $0.04 \pm 0.07$ \\
\hline 1811 & HD 188326 & 0.172 & 3 & 1.092 & $5272 \pm 40$ & $3.80 \pm 0.01$ & $-0.18 \pm 0.00$ & $5074 \pm 39$ & $3.34 \pm 0.15$ & $-0.20 \pm 0.21$ & $0.12 \pm 0.03$ \\
\hline 1812 & HD 188326 & 0.161 & 3 & 1.084 & $5272 \pm 40$ & $3.80 \pm 0.01$ & $-0.18 \pm 0.00$ & $5074 \pm 41$ & $3.34 \pm 0.16$ & $-0.20 \pm 0.21$ & $0.12 \pm 0.03$ \\
\hline 1876 & HD 212943 & 0.081 & 4 & 1.085 & $4625 \pm 67$ & $2.79 \pm 0.05$ & $-0.29 \pm 0.09$ & $4656 \pm 35$ & $2.61 \pm 0.10$ & $-0.30 \pm 0.04$ & $0.17 \pm 0.07$ \\
\hline 1893 & HD 216219 & 0.093 & 1 & 1.000 & $5628 \pm 106$ & $3.12 \pm 0.22$ & $-0.41 \pm 0.10$ & $5727 \pm 152$ & $3.36 \pm 0.50$ & $-0.39 \pm 0.21$ & $0.06 \pm 0.19$ \\
\hline 1916 & HD 219449 & 0.070 & 16 & 1.259 & $4647 \pm 75$ & $2.56 \pm 0.26$ & $-0.03 \pm 0.07$ & $4626 \pm 35$ & $2.39 \pm 0.07$ & $-0.03 \pm 0.03$ & $0.03 \pm 0.03$ \\
\hline
\end{tabular}

Notes. The literature parameters are the average from the PASTEL catalogue. Our results were obtained using the MILES library. See details in Sect. 3.2.2. 
B. Dias et al.: FORS2/VLT survey of Milky Way globular clusters. I.

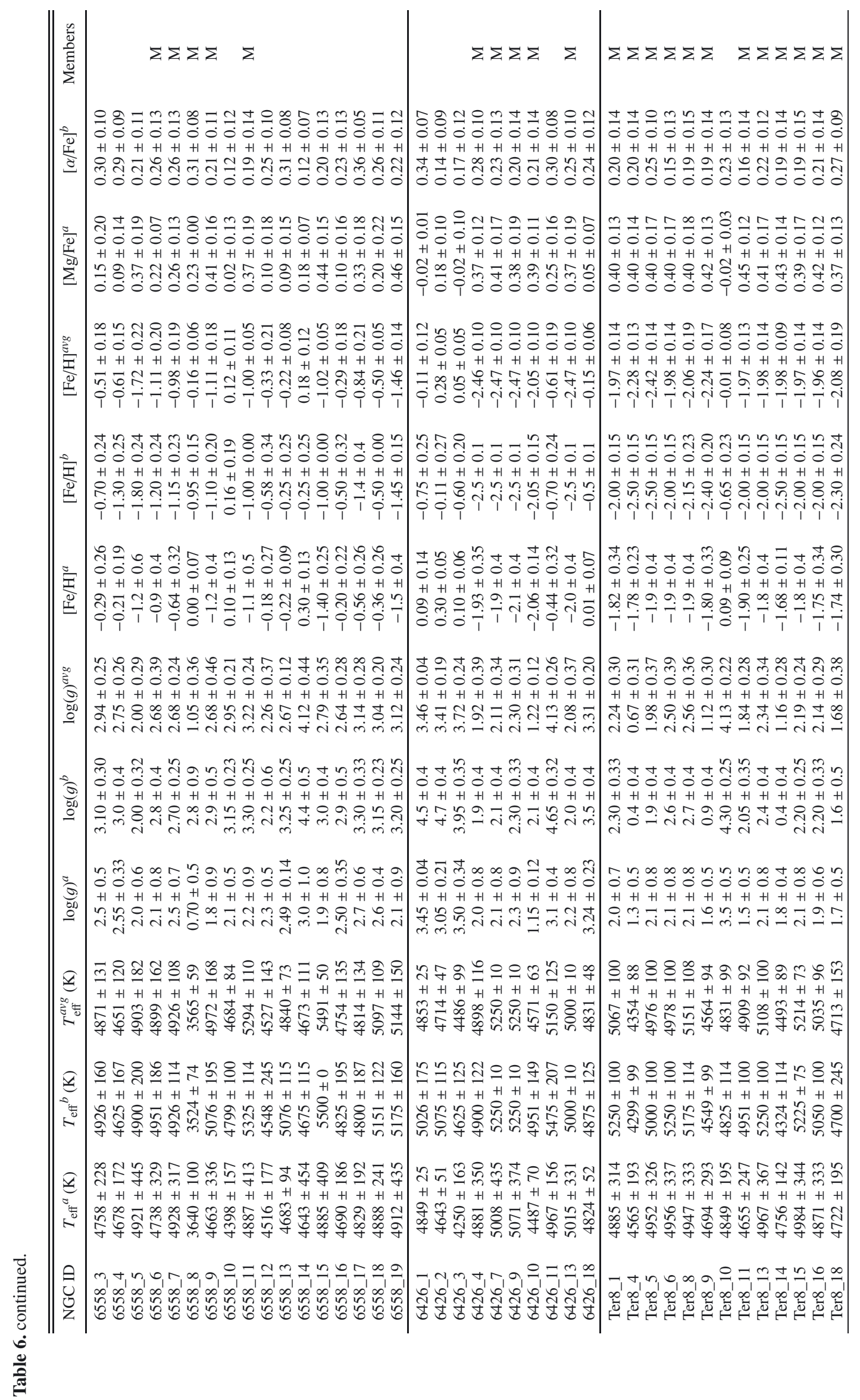

A13, page 23 of 23 